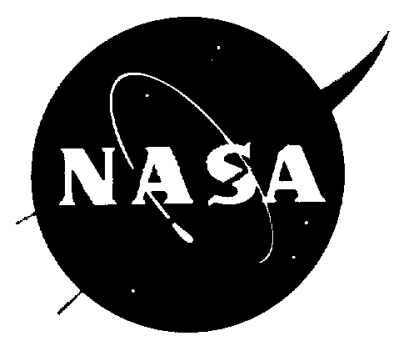

NAṠA Technical Memorandum 108479

\title{
Transport Phenomena in the Micropores of Plug-Type Phase Separators
}

M.M. Fazah

Marshall Space Flight Center $\bullet$ MSFC, Alabama 


\section{ACKNOWLEDGMENTS}

I would like to express my appreciation to my advisor, Professor Gerald Karr, for his assistance in the area of porous plug technology and fluid dynamics and to the remainder of my committee,

Professors Hugh Coleman and Clark Hawk, for their assistance and technical guidance. I also would like to thank Dr. George Schmidt for his valuable insight and guidance in the computer code development and modification. 


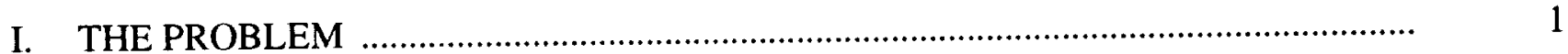
A. Introduction
B. Background
C. Statement of the Problem
D. Approach
E. Summary

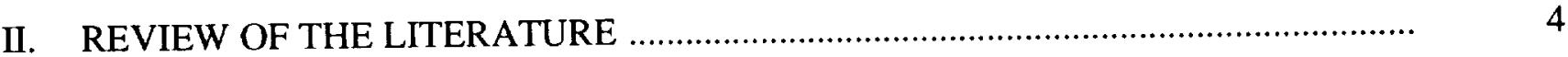
A. Introduction
B. Porous-Plug Research
C. Relevent Numerical Models
D. Summary

III. DESCRIPTION OF REFERENCE MODEL

A. Introduction

B. Physical and Numerical Model

C. Summary

IV. MODIFICATIONS TO REFERENCE MODEL

A. Introduction

B. Free Surface Boundary Condition

C. Lower Boundary Condition

D. Sidewall Boundary Condition

E. Summary

V. RESULTS AND FINDINGS

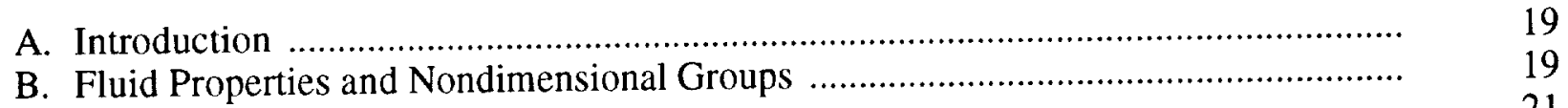

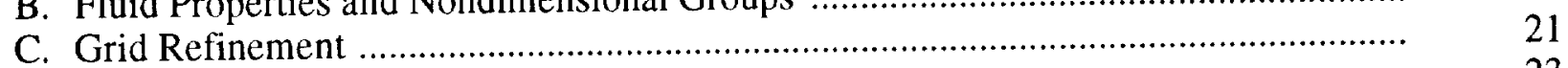

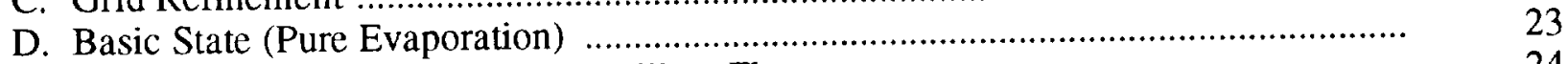

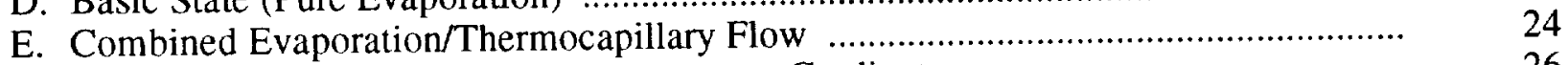

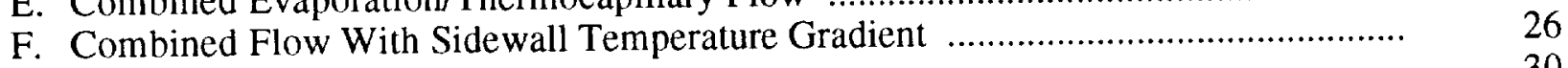

G. Summary

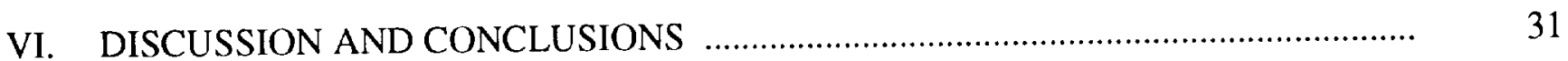
A. Introduction
B. Discussion
31
C. Conclusions 
TABLE OF CONTENTS (Continued)

D. Recommedations

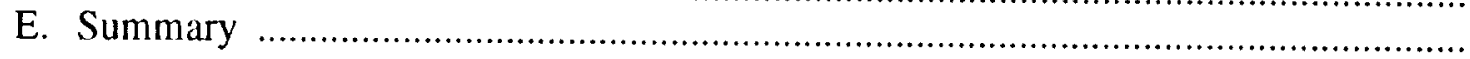

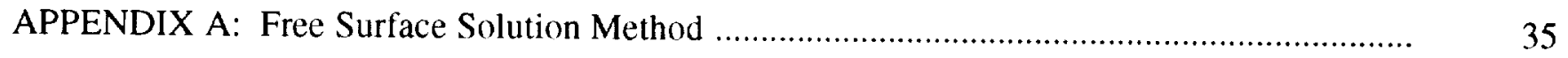

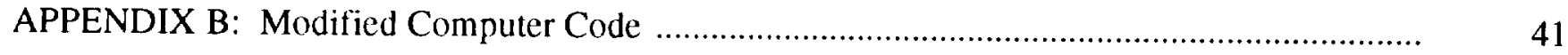

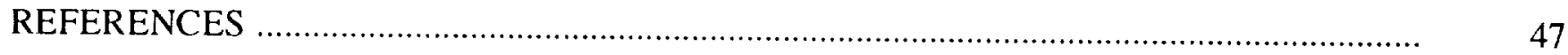




\section{LIST OF FIGURES}

Figure

Title

Page

1. Porous plug

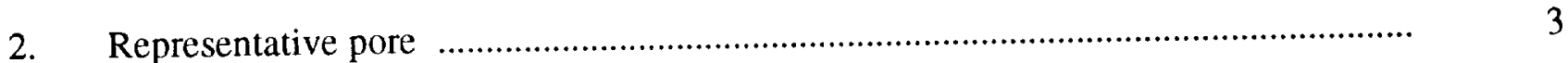

3. LAD pore schematic

4. Reference model problem domain ........................................................................

5. Free surface coordinate system ........................................................................... 12

6. Free surface shape versus pore pressure differential ................................................. 15

7. Lower boundary condition coordinate frame ………............................................... 16

8. Lower boundary velocity distribution ………........................................................ 17

9. Side boundary temperature distribution .................................................................

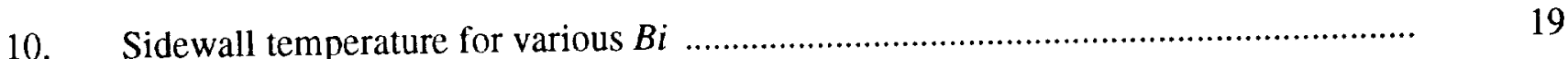

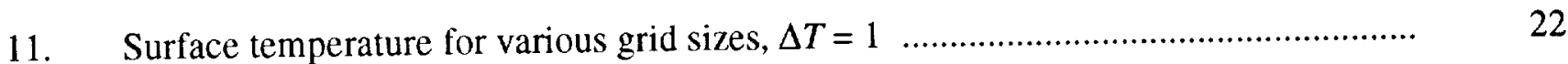

12. Basic state temperature and stream function distribution ............................................. 23

13. Basic state surface temperature distribution ............................................................. 24

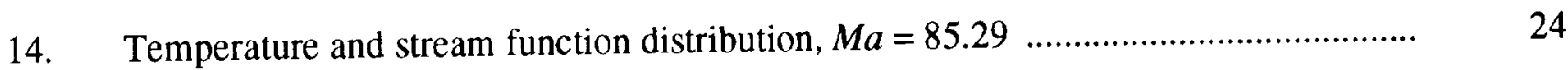

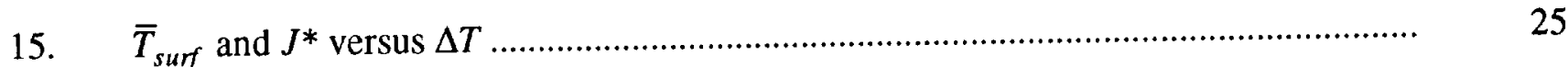

16. Surface divergence from basic state for various $\Delta T$................................................. 26

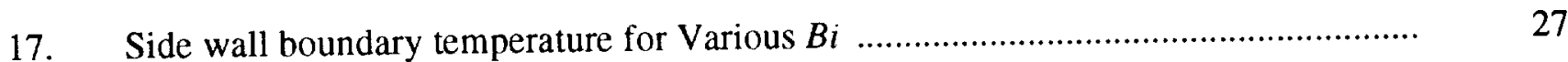

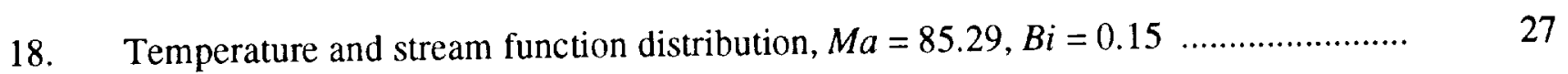

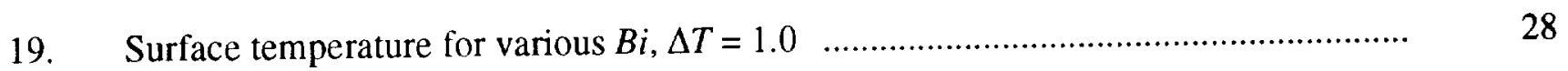

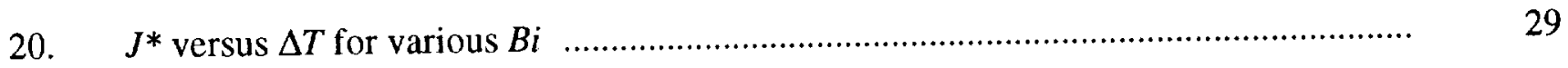

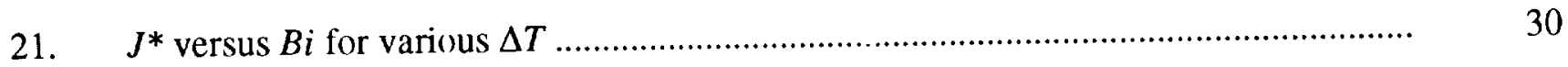




\section{LIST OF TABLES}

Table

Title

Page

1. Saturated hydrogen properties

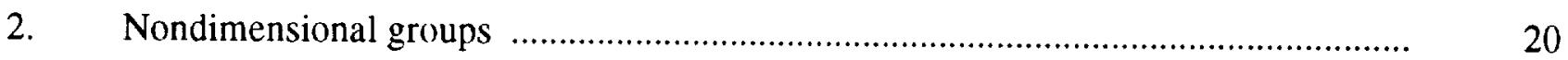

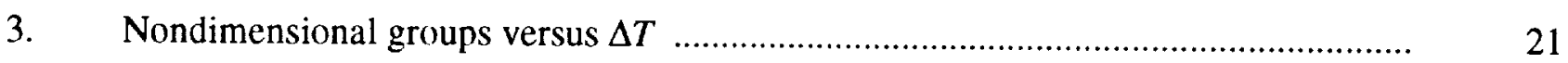

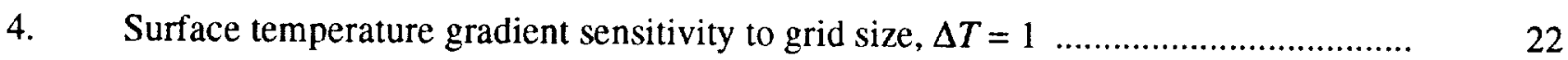

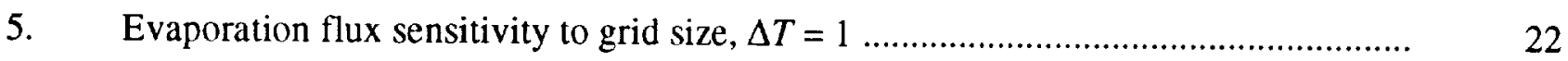

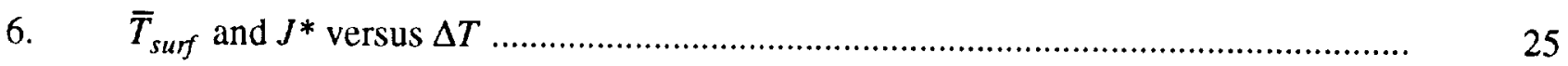

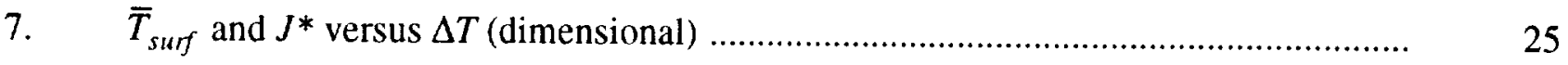

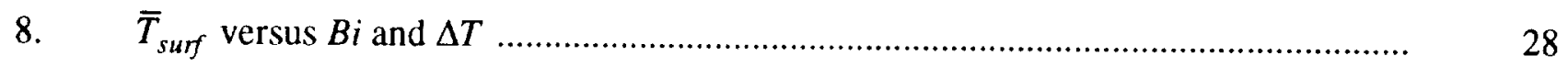

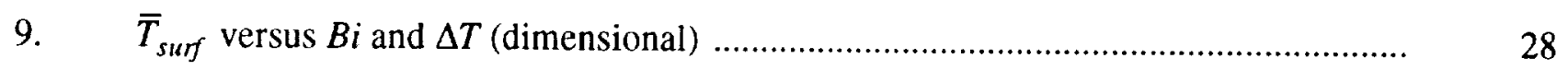

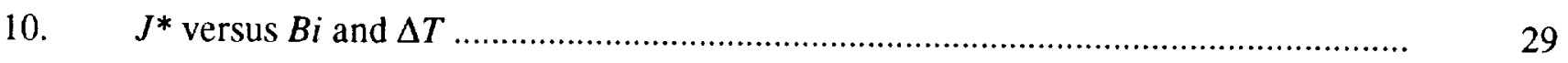

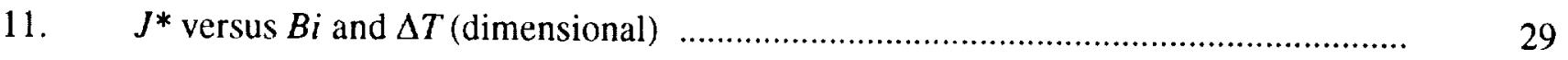




\section{LIST OF SYMBOLS}

\begin{tabular}{|c|c|}
\hline Symbol & Definition \\
\hline$a$ & pore radius \\
\hline$a_{i}$ & normalized acceleration \\
\hline$\alpha$ & coefficient of evaporation \\
\hline$\beta$ & thermal expansion coefficient \\
\hline$C p$ & specific heat \\
\hline $\mathrm{D}$ & pore diameter \\
\hline$\Delta_{i}$ & surface change \\
\hline$\Delta P$ & pressure differential \\
\hline$\Delta P_{b}$ & pore bubble point \\
\hline$\Delta T$ & liquid superheat \\
\hline$f_{\rho}$ & ratio of liquid to vapor density \\
\hline$g_{i}$ & Earth acceleration \\
\hline$\Gamma$ & boundary area \\
\hline$\gamma$ & surface tension coefficient \\
\hline$h$ & convective heat transfer coefficient \\
\hline$h$ & distance between side boundary nodes \\
\hline$j$ & nodal evaporation flux \\
\hline$J$ & pore evaporation flux \\
\hline$k$ & thermal conductivity \\
\hline$\kappa$ & surface curvature \\
\hline $\mathrm{L}$ & latent heat \\
\hline$M_{w}$ & molar weight \\
\hline$\mu$ & dynamic viscosity \\
\hline$n_{i}$ & unit normal vector \\
\hline$v$ & kinematic viscosity \\
\hline
\end{tabular}




$\begin{array}{ll}P & \text { pressure } \\ R_{g} & \text { universal gas constant } \\ \rho & \text { density } \\ t & \text { time } \\ T & \text { temperature } \\ \bar{T}_{\text {surf }} & \text { mean surface temperature } \\ u & \text { boundary 2 velocity distribution } \\ V_{i} & \text { velocity component } \\ x_{i} & \text { Cartesian coordinates } \\ \zeta & \text { thermal diffusivity }\end{array}$

Nondimensional Groups

$\begin{array}{ll}A R & \text { aspect ratio } \\ B i & \text { biot number } \\ B o & \text { bond number } \\ \mathrm{Ca} & \text { capillary number } \\ \mathrm{Cr} & \text { crispation number } \\ \mathrm{E} & \text { evaporation number } \\ \mathrm{Gr} & \text { Grashoff number } \\ \mathrm{Ma} & \text { Marangoni number } \\ \mathrm{Nu} & \text { Nusselt number } \\ \mathrm{Pr} & \text { Prandtl number } \\ \mathrm{Re} & \text { surface tension Reynolds number } \\ \mathrm{Rs} & \text { evaporation resistance } \\ \mathrm{Vr} & \text { recoil parameter }\end{array}$




\section{$\underline{\text { Subscripts }}$}

$\begin{array}{ll}b & \text { value at bubble point } \\ b & \text { value at plug solid boundary } \\ i & \text { first-order tensor index } \\ i j & \text { second-order tensor index } \\ , i & \partial / \partial x_{i} \\ , i j & \partial^{2} / \partial x_{i} \partial x_{j} \\ l & \text { value at minimum cavity temperature } \\ o & \text { value in vapor } \\ u & \text { value in tank ullage } \\ \max & \text { maximum value of variable } \\ 1,2,3,4 & \text { boundary designation } \\ s a t & \text { value at saturation point }\end{array}$

\section{Superscripts}

$\begin{array}{ll}(v) & \text { vapor } \\ (l) & \text { liquid } \\ * & \text { dimensionless parameter } \\ (s) & \text { value at surface } \\ (\text { sol }) & \text { value at solid } \\ (k) & \text { iteration number }\end{array}$





\section{TECHNICAL MEMORANDUM}

\section{TRANSPORT PHENOMENA IN THE MICROPORES OF PLUG-TYPE PHASE SEPARATORS}

\section{THE PROBLEM}

\section{A. Introduction}

This study investigates the transport phenomena within a micropore of a porous-plug trapping device. The porous plug has been used as a phase separator in liquid helium systems and can potentially be used as a pressure control device as well. In ground- or space-based storage applications, heat invariably enters the tank causing the cryogen to boil off and evaporate, thus increasing the tank pressure. The tank pressure must be controlled to prevent tank over-pressurization and structural failure and to maintain the desired thermodynamic state for the particular application. Tank venting can be accomplished easily in ground-based systems since the vapor location is predictable, namely at the top of the tank. In space-based systems where the acceleration is minimized, tank venting becomes more complex since the vapor location is unknown. Numerous studies in the past have examined both active and passive techniques for venting and tank pressure reduction. ${ }^{1}$ Active methods require complex systems that employ mechanical pumps and valves to achieve efficient venting of liquid-free vapor. Passive venting is much simpler since operation of the pressure control device is driven entirely by the pressure and temperature differential across the liquid/vapor interface and does not require the use of complex hardware.

In the past, performance of porous-plug trapping devices has been estimated by assuming that the liquid in the individual pore is static and that the heat transfer through the pore is entirely by conduction. Recent work using pore sizes in the range of the porous plug, 0.1 to 1.0 micrometers $(\mu \mathrm{m})$, have indicated that thermocapillary-and evaporation-driven convection may also be present. Therefore, the objective of this study is to investigate some of the physical parameters that influence the transport phenomena within the porous plug.

\section{B. Background}

Advanced cryogenic tankage requires the use of sophisticated insulation systems to minimize the heat into the fluid from the environment. Since the insulation cannot completely intercept the heat entering the tank, a means of rejecting this heat is required. Heat rejection through venting is one common approach.

Microgravity venting techniques studied in the past include both active and passive systems. Active systems offer more control over the venting process since the system can be designed to accommodate variable conditions and high heat rejection rates. One technique for active venting in microgravity is to apply either continuous or intermittent thrust to settle the liquid into a predetermined location within the tank, thus allowing venting of liquid-free vapor. This operation complicates mission planning by requiring vent operations to coincide with RCS thrusting operations. Settled venting has been used as the primary technique on the Saturn S-IVB stage and is currently employed on the Centaur upper stages. Another active venting method that has been studied extensively is the thermodynamic 
vent system (TVS). 23 The TVS provides the combined function of heat extraction and mixing of the liquid and vapor, i.e., thermal destratification. Heat is rejected by passing a small amount of liquid through a Joule-Thompson (J-T) valve and a heat exchanger. When the liquid is passed through the J-T valve, it is flashed into a two-phase mixture, which results in a temperature decrease due to the pressure drop across the valve. This cooler fluid is then used as a heat sink by passing it through a heat exchanger which extracts energy from the circulating bulk liquid. The two-phase flow is superheated in the heat exchanger and subsequently vented as a gas. TVS concepts are highly efficient and can be designed to reject large amounts of heat, but they are relatively complex, heavy, and expensive.

Passive systems based on porous-plug phase separation are much simpler and rely on a pressure differential and the thermodynamic properties of the cryogen to reject energy from the system. Porous plugs have been studied and tested previously with superfluid helium. ${ }^{4-6}$ These devices mainly consist of a sintered metal or fine screen mesh and are difficult to analyze due to irregularly shaped flow passages and inherent nonuniformities in the manufacturing process. These devices exploit the fluid's surface tension to form a phase boundary between the liquid and the vapor in the microscopic passages within the sintered metal or screens. These passages typically range from 0.1 to $1.0 \mu \mathrm{m}$ in diameter. Evaporation takes place across the liquid/vapor boundary to reject heat. An advanced porous plug that has been designed and tested recently ${ }^{7}$ uses an advanced manufacturing process to fabricate a plug with multiple uniform cylindrical passages running parallel to its axis of symmetry. This type of design is easier to analyze due to its simple geometry. Figure 1 shows a typical wetted porous plug that is mounted in the wall of a cryogenic dewar or tank.

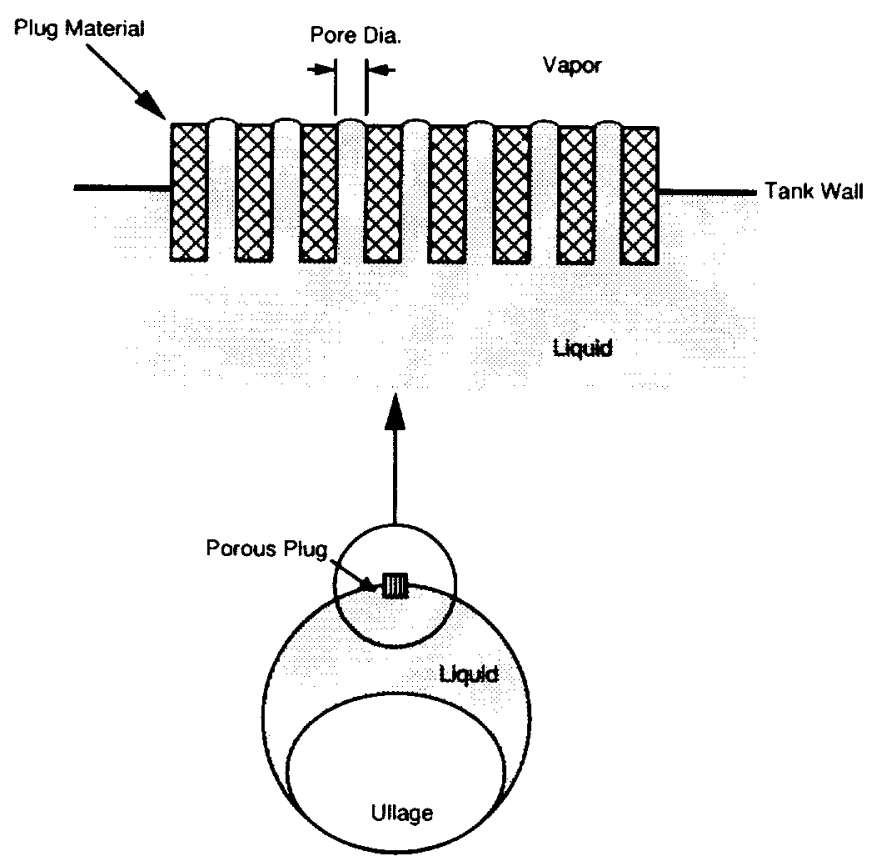

Figure 1. Porous plug.

During nominal operations the plug provides a means of venting the tank and controlling tank pressure without expelling liquid across the pore surface. One means of passively controlling tank pressure and rejecting heat from a liquid is through evaporation across the liquid-vapor interface in a porous plug. If the liquid vapor pressure is greater than the ullage pressure, the liquid is superheated with respect to its saturated state. In order for the liquid to reach saturation, the liquid must reject heat or energy. This rejection is accomplished through evaporation within the porous plug. The porous plug can 
perform this function regardless of whether liquid or vapor is in contact with the internal surface of the plug. Past studies have shown that for the sintered porous plugs tested, there are three distinct observable flow regimes observed. ${ }^{4}$ In the first regime, the pore is fully wetted, i.e., filled with liquid, evaporation occurs across the interface, and the liquid assumes an equilibrium position as shown in figure 2 . The liquid/vapor interface residing on the vent side of the plug provides an evaporation site that transfers energy from the tank fluid and enables pressure control. The geometry of this interface is dictated by the jump momentum balance and includes the effects of pressure-, temperature-, and evaporation-induced recoil. In the second regime, the pore is dry and the vapor flow through the pore is laminar. In the third regime, the plug is also dry, as was the second regime, but the vapor flow is turbulent. This study concentrates on the wetted case, namely regime one.

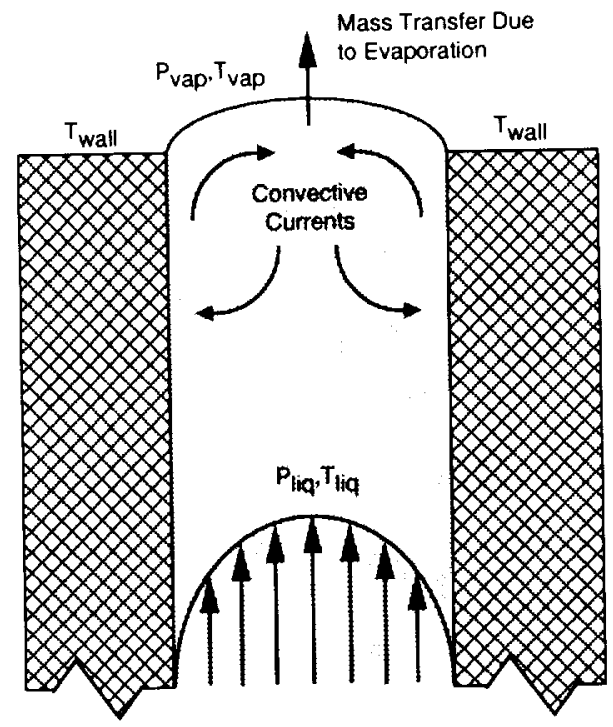

Figure 2. Representative pore.

An important aspect of the pore is the ability to sustain a pressure differential across the interface. As shown in figure 1 , the pressure differential is caused by the tank pressure being greater than the vapor pressure on the exterior of the pore. The maximum pressure that the pore can sustain across the interface is commonly called the "bubble point" pressure and is theoretically described by the LaPlace Young equation:

$$
\Delta P_{b}=\frac{4 \gamma}{D}
$$

where $D$ is the pore diameter. As long as the pressure difference across the plug is equal to or less than the value given by equation (1), the liquid will remain in the plug. Unlike sintered metal constructed porous plugs where the effective pore diameter is not constant and little control over the pore size is available, a porous plug with uniform cylindrical pores can be designed for a particular fluid and desired bubble point.

\section{Statement of the Problem}

In the wetted case (regime one) the heat rejection mechanism is much more complicated than in regimes two or three since the fluid dynamics in the liquid are more difficult to analyze than the vapor 
flow through a pore. Heat must be rejected by evaporation across the interface. In the liquid, convection is driven by sidewall heating, evaporation, and thermocapillary effects. Most porous plug studies have assumed superfluid helium as the working fluid and only recently has hydrogen been discussed.

In the previous studies, fluid dynamics within the pore could not be analyzed due to the complicated nature of the flow passages. Assumptions and simplifications were made to allow some understanding of the fundamentals involved. The uniform pore design allows the use of computational fluid dynamics (CFD) techniques and can provide a more detailed investigation of the physical parameters that affect the transport phenomena within the pore.

The objective of this study is to investigate the transport phenomena within the pores of a porous-plug trapping device. The effect of temperature differential across the interface and sidewall heating conditions has been examined.

\section{Approach}

The approach used to investigate the transport phenomena in a pore was to use CFD to model a representative pore. A reference finite element model developed to analyze a liquid acquisition device (LAD) pore ${ }^{8}$ described in section III, has been modified to account for the physical differences of the LAD and porous plug. The modified model is then used to investigate the influence of various physical parameters on the flow field within the pore and the mass transfer or evaporation across the pore interface.

\section{E. Summary}

Pressure control for space-based systems becomes more complicated than ground-based due to the uncertainty of the ullage location. Pressure control devices that achieve liquid-free venting have been studied in the past and include the porous-plug phase separator. Past studies had identified three flow regimes within the porous plug through experimentation. The complicated geometry and flow passages of the porous plugs have precluded detailed analysis until uniform cylindrical microscopic flow passages were able to be manufactured through advanced processes. This study numerically investigates the transport within and across a single cylindrical pore of porous plug that is wetted with liquid hydrogen.

\section{REVIEW OF THE LITERATURE}

\section{A. Introduction}

The concept of phase separation using a porous media or plug has been studied in the past by several researchers. Past research is discussed in this section, namely, work that has been done using superfluid helium or He II, normal fluid helium, and initial work that has been done for liquid hydrogen using a porous plug as the phase separation device. Other work that pertains to the phase separation across an LAD pore is also discussed. The porous plug and LAD applications are different, but the governing equations for the flow within and across the LAD are also valid for the porous-plug flow field. 


\section{B. Porous-Plug Research}

As mentioned earlier, most of the past studies of sintered metal porous plug operation have been based on He II. Cooling requirements for experimental electronics have driven the need for He II phase separation devices. Karr and Urban ${ }^{4}$ and Frederking et al..$^{5}$ investigated using the porous plug to modulate helium vapor flow for the cooling of an experiment. Karr and Urban used an $\mathrm{Al}_{2}-\mathrm{O}_{3}$ ceramic plug with a nominal $5-\mu \mathrm{m}$ diameter pore size to evaluate both the steady and unsteady porous plug operation with helium. Steady-state data were obtained by measuring the mass flow rate through the plug as a function of the downstream pressure, and it was observed that the relation of $\dot{m}$ to $\Delta P$ shows two distinct regimes, characterized by a change in slope of the curve. To experimentally evaluate the unsteady operation of the plug, a heater was installed downstream of the plug and used to modulate the vapor flow across the plug. This technique was effective in supplying cold helium vapor on demand. Frederking et al. used a flow control plate and shutter assembly to increase or decrease the evaporative area of the porous plug by opening or closing the shutter. This technique was also effective in regulating the supply of cold vapor. In both studies the standard two-fluid model for He II is used to theoretically characterize the mass flow through the pore.

Hendricks and Karr, ${ }^{679}$ have investigated the flow regimes previously identified in reference 4 and the transition points between the regimes. In reference 6 , a 316 stainless steel plug with an $0.5-\mu \mathrm{m}$ filtration grade was used to experimentally examine the transition between the lower and upper regimes. The lower regime was proposed to be defined when the liquid/vapor interface is downstream of the plug and the plug is filled with liquid. It was shown that the regime, transition occurs at the critical mass flow rate, $\dot{m}_{\text {crit }}$. In the upper regime, it was proposed that the liquid/vapor interface retreats into the plug, thus filling the pores with vapor. One explanation for this phenomena is that as the heat flux on the plug is increased, there comes a point where the liquid can no longer transfer the heat to the interface; therefore, the interface must move such that the energy is balanced.

Schotte ${ }^{10}$ examined He II phase separation using a narrow annular slit instead of the sinter metal porous plug. As with references 6,7 , and 9 , Schotte measured the relation of $\dot{m}$ to $\Delta P$ and observed the same transition occurring at the critical mass flow rate. Using the two fluid model, Schotte was able to show a good comparison with the experimental data. This was probably due to the uniformity and simplicity of the experiment geometry.

Recently, utilization of a porous plug with fluids other than He II have been evaluated. Hendricks and Dingus ${ }^{11}$ used normal fluid $\mathrm{He}$ or ${ }^{4} \mathrm{He}$ to evaluate the bubble point of a porous plug constructed of $\mathrm{Fe}_{2}-\mathrm{O}_{2}$ or jewelers rouge, as a function of the ${ }^{4} \mathrm{He}$ liquid temperature. The test data showed that the observed bubble point for ${ }^{4} \mathrm{He}$ decreased with increasing liquid temperature; therefore, the liquid must be precooled prior to entering the plug to prevent retention loss. Of more direct interest is work done by Hendricks and Dingus ${ }^{12}$ on using a porous plug in a liquid hydrogen system. The porous plug used consisted of multiple uniform pores that were parallel with the plugs axis of symmetry. The advantage is that it allowed for more detailed analysis of the flow field within the individual pore since the geometry can be modeled more accurately. To evaluate the evaporation across the interface of the pore, a simple one-dimensional fin model was used. The analysis assumed that heat is transferred to the interface by solid conduction through the plug material and that liquid convection within the pore interior is negligible. 


\section{Relevant Numerical Models}

Sasmal and Hochstein ${ }^{13}$ developed a finite volume model to predict the transient behavior of the thermocapillary convection within a two-dimensional cavity with a deforming interface. The fluid motion, thermocapillary convection, is driven by a variation in surface tension due to an imposed temperature gradient. In this case, the left boundary was held at a higher temperature than the right bound ary and zero heat transfer was specified across the free surface and the lower boundary. Since the condition of zero heat transfer across the surface was imposed, no evaporation or interfacial mass transfer was considered. Sasmal and Hochstein modeled various free-surface geometric configurations including flat surface; acute contact angle, i.e., wetting fluid; and obtuse contact angle, i.e., nonwetting fluid. The obtuse contact angle case is more representative of the problem considered in this study since it represents the surface due to the pressure differential between the tank ullage and the exterior of the plug. Their analysis showed that the free surface rises along the cold wall and falls along the hot wall. This phenomena was most likely due to the fluid being driven away from the hot and toward the cold boundary by the thermocapillary stress condition.

Schmidt ${ }^{8}$ developed a finite element model to investigate the steady-state behavior of the flow about an evaporating or condensing LAD pore, while accounting for thermocapillary convection. The problem domain consisted of a two-dimensional cavity having a curved free surface with an acute contact angle. A temperature gradient was imposed across the free surface to examine the influence of interfacial mass transfer and thermocapillary convection on the liquid retention of the pore. This model is discussed in more detail in section III. One of the more significant findings by Schmidt was that thermocapillary convection arising from condensation can cause a retention loss, while thermocapillary convection arising from evaporation causes a decrease in the surface curvature and, therefore, tends to make the interface remain stable.

\section{Summary}

In this section, previous experimental and analytical work pertaining to the porous plug trapping devices is discussed. Studies which concentrated on He II, normal He, and preliminary work with liquid hydrogen was summarized. Also, numerical models that are relevant to the problem addressed in this study are discussed.

\section{DESCRIPTION OF REFERENCE MODEL}

\section{A. Introduction}

As shown in section II, Schmidt ${ }^{8}$ has developed a two-dimensional finite element model of evaporation or condensation within an LAD pore. The model accounts for thermocapillary convection at the pore interface and the associated convection within the pore along with the mass transfer at the interface. The pore's free surface is modeled with respect to the physical parameters at the interface, such as temperature, pressure, and fluid velocity. This model is used as the basis for the analysis of this study. 


\section{B. Physical and Numerical Model}

The physical domain of the reference model approximates that of the cross section of a single two dimensional pore of the LAD. The cross section, shown in figure 3, shows an individual interface or meniscus attached to individual circular screen wires.

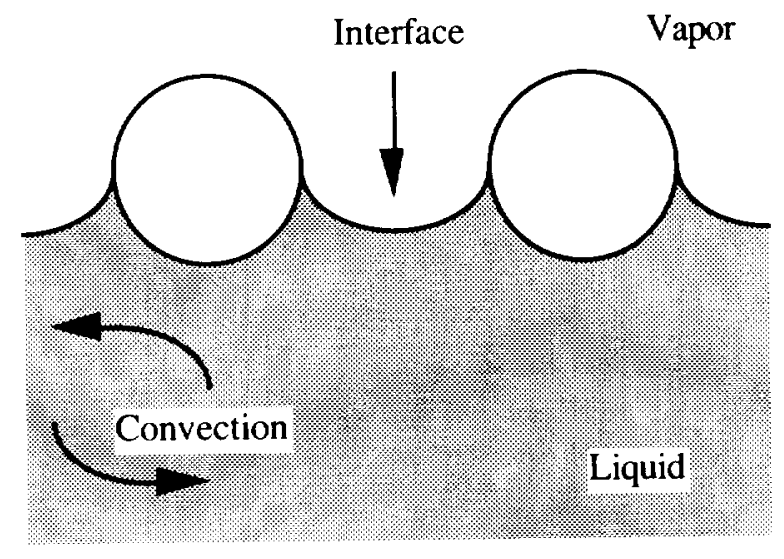

Figure 3. LAD pore schematic.

The boundary conditions for this representation are complex. The curved surface representing the screen wires and the free flow or unspecified flow boundaries at the left and right side of the domain would make this problem complicated to solve and, therefore, would require a much more sophisticated numerical model. In order to simplify the problem, several assumptions were made to obtain the problem domain. The first assumption is that the interface diameter is small compared to the screen wire diameter; therefore, the curved screen boundaries can be approximated as flat. A second simplifying assumption is that the sidewall boundary conditions are no-slip. Figure 4 shows the problem domain that is used in the reference model.

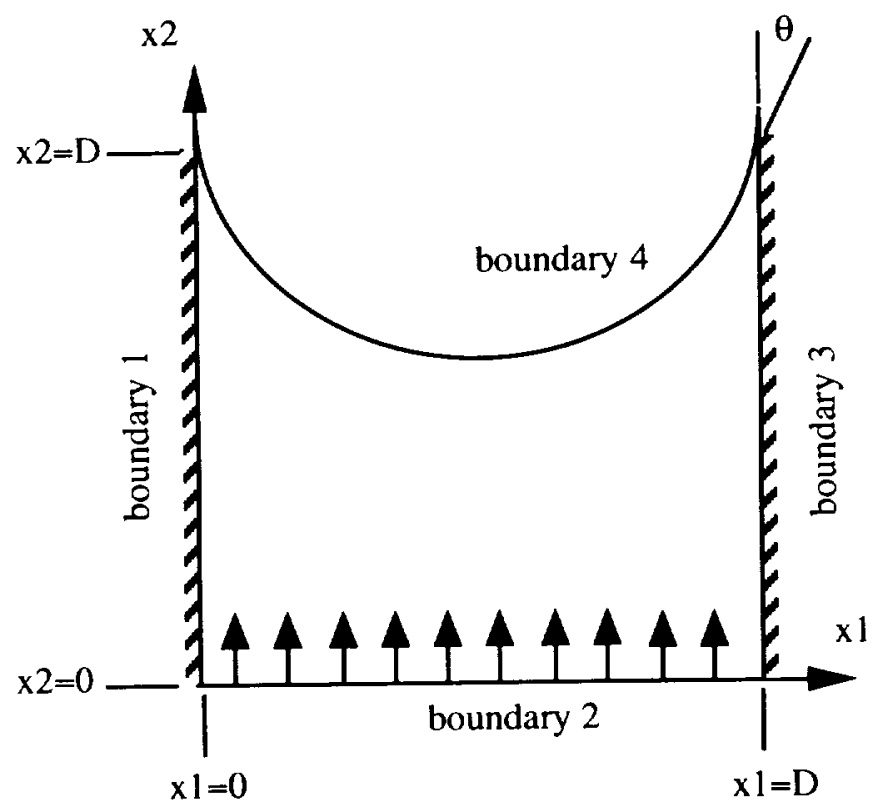

Figure 4. Reference model problem domain. 
The governing equations for the reference model are based on constant properties and derived from the continuity, momentum, and energy equations for an incompressible fluid, as shown below.

$$
\begin{gathered}
V_{i, i}=0 \\
\rho \frac{\partial V_{i}}{\partial t}+\rho V_{j} V_{i, j}+P_{d, i}-\tau_{i j, j}-\rho \beta\left(T-T_{l}\right) g_{i}=0 \\
\frac{\partial T}{\partial t}+V_{j} T_{, j}-\zeta T_{, j j}=0
\end{gathered}
$$

where

and

$$
\left.\beta=-\frac{1}{\rho} \frac{\partial \rho}{\partial T}\right)_{P}
$$

$$
\tau_{i j}=\mu\left(V_{i, j}+V_{j, i}\right)
$$

The Boussinesq approximation is used in the momentum equation (3) to account for buoyant forces. This also restricts the thermal dependence of density as a body force.

A fourth governing equation defines the free surface shape due to the flow and temperature field characteristics. The normal component of the jump momentum balance on the surface yields a relation that represents the surface curvature and is given by:

$$
\gamma \kappa=\left(P^{(v)}-P_{d}-P_{h}\right)+\frac{j^{2}}{\rho}\left(f_{\rho}-1\right)+2 \mu V_{i, j} n_{j} n_{i},
$$

where $\mathrm{K}$ is the surface curvature, $P^{(v)}$ is the vapor pressure, $P_{d}$ is the liquid dynamic pressure, $P_{h}$ is the hydrostatic pressure, $j$ is the evaporation flux, $f_{\rho}$ is the ratio of the liquid density to vapor density, and term $2 \mu V_{i, j} n_{j} n_{i}$ represents the viscous stress on the surface. dimensions.

The surface curvature can also be defined using the expression for the radius of curvature in two

$$
\kappa=-\frac{1}{y_{, x}^{(s)}} \frac{d}{d x}\left\{\left[1+\left(y_{, x}^{(s)}\right)^{2}\right]^{-1 / 2}\right\} .
$$

Two additional relations are used, one to account for thermocapillarity and the other to account for the mass transfer across the interface. In order to account for the thermocapillary effect of the fluid, i.e., the thermal dependence of surface tension, a linear relation of surface tension as a function of temperature is used:

$$
\gamma=\gamma_{l}-\left|\frac{\partial \gamma}{\partial T}\right|\left(T-T_{l}\right)
$$


To account for the evaporation across the interface, the relation given below ${ }^{14}$ is used to relate the mass transfer to the liquid temperature and its thermophysical properties:

$$
j=\alpha\left(\frac{R_{g} T}{2 \pi M_{W}}\right)^{1 / 2}\left(\rho-\rho_{o}\right),
$$

where $\alpha$ is the coefficient of evaporation that estimates the fraction of impinging liquid molecules that do not evaporate, i.e., some of the molecules rebound off the surface rather than evaporating. The maximum evaporation flux occurs when $\alpha=1$. The universal gas constant and the molar weight of the vapor are given by $R_{g}$ and $M_{w}$, respectively. The density difference in equation (10) represents the divergence from saturation to a superheated state corresponding to temperature $T$. It was shown ${ }^{8}$ that the mass flux can be related to a temperature difference across the interface and takes the form of:

$$
j=\frac{\alpha \rho_{o}^{(v)} L}{T_{o}^{3 / 2}}\left(\frac{M_{w}}{2 \pi R_{g}}\right)^{1 / 2}\left(T-T_{o}\right)
$$

All the aforementioned equations and relations are then nondimensionalized using the following scaling relations:

$$
\begin{array}{cc}
x_{i}=D x_{i}^{*}, & V_{i}=\frac{v}{D} V_{i}^{*}, \\
t=\frac{D^{2}}{v} t^{*}, & g_{i}=a_{i} g_{o}, \\
\sigma_{i j}=\frac{\rho v^{2}}{D^{2}} \sigma_{i j}^{*}, & P=\frac{\rho v^{2}}{D^{2}} P^{*}, \\
j=\frac{\rho v}{D} j^{*}, & T=T^{*}|\Delta T|+T_{0} .
\end{array}
$$

Using the scaling relations given in equation (12), the following nondimensional governing equations are obtained:

$$
\begin{gathered}
V_{i, i}^{*}=0, \\
\frac{\partial V_{i}^{*}}{\partial t^{*}}+V_{j}^{*} V_{i, j}^{*}+P_{d, i}^{*}-\tau_{i j, j}^{*}-G r a_{i} T^{*}=0 \\
\frac{\partial T^{*}}{\partial t^{*}}+V_{j}^{*} T_{, j}^{*}-\frac{1}{\operatorname{Pr}} T_{, j j}^{*}=0,
\end{gathered}
$$

where

$$
G r=\frac{D^{3} g \beta|\Delta T|}{v^{2}}, \quad \text { Grashoff number }
$$




$$
\operatorname{Pr}=\frac{v}{\zeta} \cdot \quad \text { Prandtl number }
$$

Using equation (7), the equation for the interfacial curvature is given by:

$$
\kappa^{*}=\frac{B_{o} y^{(s)^{*}}-C a\left(P_{d}^{*}-P^{(v)^{*}}\right)+V_{r} T^{* 2}+2 C a V_{i, j}^{*} n_{j} n_{i}}{1-C r T^{*}},
$$

where,

$$
\begin{array}{cc}
B o=\frac{\rho g D^{2}}{\gamma_{i}}, & \text { bond number } \\
C a=\frac{\rho v^{2}}{\gamma_{l} D}, & \text { capillary number } \\
V_{r}=\frac{C a\left(f_{\rho}-1\right)}{R s^{2}}, & \text { recoil parameter } \\
C r=\frac{1}{\gamma}\left|\frac{\partial \gamma}{\partial T}\right||\Delta T|, & \text { crispation number }
\end{array}
$$

and from equation (8),

$$
\kappa^{*}=-\frac{1}{y_{, x}^{(s)^{*}}} \frac{d}{d x^{*}}\left\{\left[1+\left(y_{, x}^{(s)^{*}}\right)^{2}\right]^{-1 / 2}\right\} .
$$

Using equation (11), the relation for the mass transfer across the interface becomes:

$$
j^{*}=\frac{T^{*}}{R s},
$$

where

$$
R s=\frac{f_{\rho} v T_{o}^{3 / 2}}{\alpha D L|\Delta T|}\left\{\frac{2 \pi R_{g}}{M_{W}}\right\}^{1 / 2} \quad, \quad \text { evaporation resistance }
$$

Boundary conditions must be imposed on the four boundaries shown in figure 4, the right and left sides and the upper and lower surfaces. Making the assumption of no-slip and impermeability on both the left and right sides, and making the further assumption that the sidewall temperature is specified and constant, the conditions on boundaries 1 and 3 are defined, namely:

$$
V_{1}^{*}=0
$$




$$
V_{2}^{*}=0
$$

and

$$
T^{*}=T_{1} \text {. }
$$

The boundary conditions on the upper and lower surfaces are more complex. The lower surface must satisfy the restriction that the flow entering or exiting the cavity through the lower surface be uniform and equal to the flow leaving or entering the cavity through the upper surface due to evaporation or condensation. Also, the temperature on the lower surface is assumed to be constant and equal to the side boundary temperature. The lower boundary conditions are:

$$
\begin{gathered}
V_{1}^{*}=0, \\
V_{2}^{*}=\frac{1}{\Gamma_{2}} \int_{\Gamma_{4}} \frac{T^{*}}{R s} d \Gamma_{4},
\end{gathered}
$$

and

$$
T^{*}=T_{1} \text {. }
$$

For the upper free surface, one must consider the normal and tangential stress conditions due to the surface curvature and the thermocapillarity, respectively, and, in addition, one must account for liquid ejected from or deposited onto the surface due to evaporation or condensation. The upper boundary conditions are:

$$
\begin{gathered}
\tau_{i j}^{*} n_{j}=-\operatorname{Re} T_{, i}^{*}=-\frac{M a}{\operatorname{Pr}} T_{, i}^{*}, \\
V_{2}^{*}=\frac{1}{n_{2}}\left(\frac{T^{*}}{R s}-V_{1}^{*} n_{1}\right), \\
T_{, i}^{*} n_{i}=-N u T^{*},
\end{gathered}
$$

where

$$
\begin{aligned}
& \operatorname{Re}=\frac{\left|\frac{\partial \gamma}{\partial T}\right| D|\Delta T|}{\rho v^{2}}, \text { surface tension Reynolds number } \\
& M a=\frac{\left|\frac{\partial \gamma}{\partial T}\right| D|\Delta T|}{\mu \zeta}, \text { Marangoni number }
\end{aligned}
$$

and

$$
N u=\frac{h D}{k^{(l)}}, \quad \text { Nusselt number . }
$$

An alternate definition for the Nusselt number, which gives the rate of latent heat transfer relative to the heat conduction is termed the mass transfer Nusselt number and is given in equation (38). It 
should be noted that in reference 8 , equations (37) and (38) are referred to as the convective Biot number, $B i_{c}$, and the mass transfer Biot number, $B i_{m}$, respectively.

$$
N u=\frac{1}{R s E}, \quad \text { mass transfer Nusselt number }
$$

where the nondimensional group, $E$, shown in equation (38) that was not previously defined is given by:

$$
E=\frac{k|\Delta T|}{\rho v L}, \quad \text { evaporation number . }
$$

As discussed in reference 8, both representations of the Nusselt number, equations (37) and (38), are equivalent in terms of defining the temperature distribution along the surface.

The surface solution, $y^{(s)}$ is determined by equating equations (18) and (23) and solving using the following boundary conditions:

$$
\begin{array}{cc}
y^{(s)^{*}}=1, & \text { at } x l=0 \text { and } 1, \\
\left|\frac{d y^{(s)^{*}}}{d x^{*}}\right|=\tan (\pi / 2-\omega), & \text { at } x l=0 \text { and } 1 .
\end{array}
$$

\section{Summary}

The reference model described in this section solves for the flow field and thermal profile in a simplified liquid acquisition device pore. The model employs a finite element numerical method to solve the governing equations and boundary conditions for velocity, temperature, pressure, and surface position. The model accounts for thermocapillary and evaporation or condensation-driven convection within the cavity.

\section{MODIFICATIONS TO REFERENCE MODEL}

\section{A. Introduction}

The problem considered in this study, the porous plug, is similar in many ways to the problem solved using the reference model ${ }^{8}$ Many of the assumptions still apply, namely, incompressibility, constant properties, and limiting the domain to two dimensions. Therefore, the governing equations are valid, i.e., continuity, momentum, and energy. Some of the boundary conditions of the domain must be modified to accurately model the problem addressed in this study.

The modifications that are required are the calculation of the free surface shape, the specification of the fully developed flow condition on the bottom boundary, and the ability to apply a temperature gradient on the side boundary as well as an isothermal boundary. The aspect ratio of the problem is also increased from one to two, but is considered minor and will not be discussed in detail. 


\section{B. Free Surface Boundary Condition}

As shown in section I, the free surface for a fully wetted porous plug differs from that of the LAD pore due to the direction of the pressure differential across the interface. In the LAD application the ullage pressure is greater than that of the liquid; therefore, the pressure differential tends to drive the ullage and the interface into the liquid. In the fully wetted porous plug application, the liquid pressure is greater than the ullage pressure; therefore, the reverse is true, namely, the pressure differential tends to drive the liquid and interface into the ullage. This fundamental difference in the direction of the $\Delta p$ requires that the surface solution be modified. Although the surface solution must be modified, the tangential stress condition, i.e., the thermocapillary effect, is still valid.

In order to define the modified surface, a change in the coordinate system is required. The coordinate system used in the free surface solution is shown in figure 5:

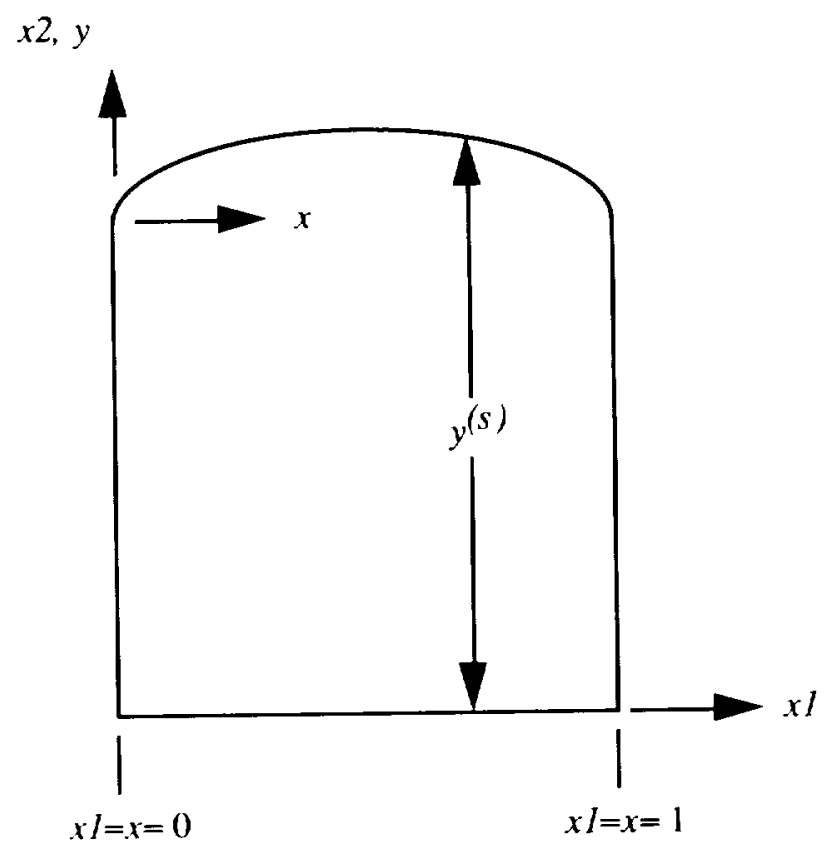

Figure 5. Free surface coordinate system.

where $x 1=x, y=y^{(s)}-x 2$ and $y^{(s)}$ is the $y$ coordinate of the surface at each $x$. In order to calculate the free surface, equations (18) through (23) are used. Expanding the derivative in the right-hand term, equation (23) can be written as:

$$
\kappa^{*}=y_{. . \alpha}^{*}\left\{\left[1+\left(y_{, x}^{*}\right)^{2}\right]^{-3 / 2}\right\}
$$

Equating equations (18) and (42) gives:

$$
y_{. x x}^{*}\left\{\left[1+\left(y_{. x}^{*}\right)^{2}\right]^{-3 / 2}\right\}=\frac{B_{o} y^{(s)^{*}}+\Pi}{1-D},
$$


where:

and

$$
\Pi=-C a\left(P_{u}^{*}+P_{d}^{*}-P^{(v)^{*}}\right)+V_{r} T^{* 2}
$$

$$
D=1-\operatorname{Cr} \bar{T}^{*}
$$

In equation (44), the viscous stress term, $2 C a V_{i, j}^{*} n_{j} n_{i}$, is dropped since its influence on the surface curvature is negligible ${ }^{8}$ and the term $P_{u}^{*}$, the tank ullage pressure, is added to account for the higher liquid pressure in the porous plug.

Rearranging equation (43) and dropping the * notation, the following second-order, nonlinear differential equation is obtained:

$$
y_{, x x}-\left(\frac{B o y^{(s)}+\Pi}{1-D}\right)\left[1+\left(y_{, x}\right)^{2}\right]^{3 / 2}=0
$$

The boundary conditions on equation (46) consist of a Dirchlet and Neumann condition. The Dirichlet condition is that at $x=0$ and $x=1, y=0$. This is commonly referred to as a pinning condition. Unlike an LAD, the boundary conditions at the end point are independent of the fluid contact angle. The Neumann condition restrains the slope of the interface at the center line to zero. This also implies that the interface is symmetrical about the center line. obtained:

In order to solve for the interface shape, a finite difference representation of equation (46) is

$$
\frac{y_{i+1}-2 y_{i}+y_{i-1}}{h^{2}}-\left(\frac{B o y_{i}+\Pi}{1-D}\right)\left[1+\left(\frac{y_{i+1}-y_{i-1}}{2 h}\right)^{2}\right]^{3 / 2}=0 \text {, }
$$

where $h$ is equal to the separation distance of the nodes.

Equation (47) is solved using Newton's method for a nonlinear series ${ }^{15}$ to converge on a solution for the independent variable $y$. Newton's method uses the following iteration algorithm:

where:

$$
y_{i}^{(k)}=y_{i}^{(k-1)}-\Delta_{i}^{(k-1)},
$$

$$
\Delta_{i}^{(k-1)}=\left(J_{i j}^{(k-1)}\right)^{-1} F_{j}^{(k-1)}
$$

and where $J_{i j}$ is defined as the Jacobian of the root vector, $F_{j}$.

Once both the Jacobian and the root equations are determined, the surface solution is found by iteration. Starting with an initial guess for $y_{i}$, the value of $\Delta_{i}^{(k-1)}$ is determined and a new value of $y_{i}$ is obtained using equation (48). This process is repeated until, $y_{i}^{(k)}-y_{i}^{(k-1)} \leq s t o l$, where stol is the surface convergence tolerance and is equal to 0.001 in this study. 
Recalling equation (46), it is seen that the nondimensional terms $C a, V_{r}$, and $C r$ are defined by the fluid under consideration, and the values of dynamic pressure and temperature are determined from the solution of the fluid governing equations. The parameter that can be specified directly is $\Delta P^{*}=P_{u}^{*}-P^{()^{*}}$. This parameter can be used to investigate the influence of the differential pressure across the pore.

One limitation of this surface model is that as the $\Delta P^{*}$ approaches $\Delta P_{b}^{*}$, the bubble point of the pore. the solution is indeterminate. This limitation occurs as the slope of the surface at $x=0$ and 1 approaches infinity. Figure 6 shows the effect of $\Delta P^{*}$ on the free surface shape.

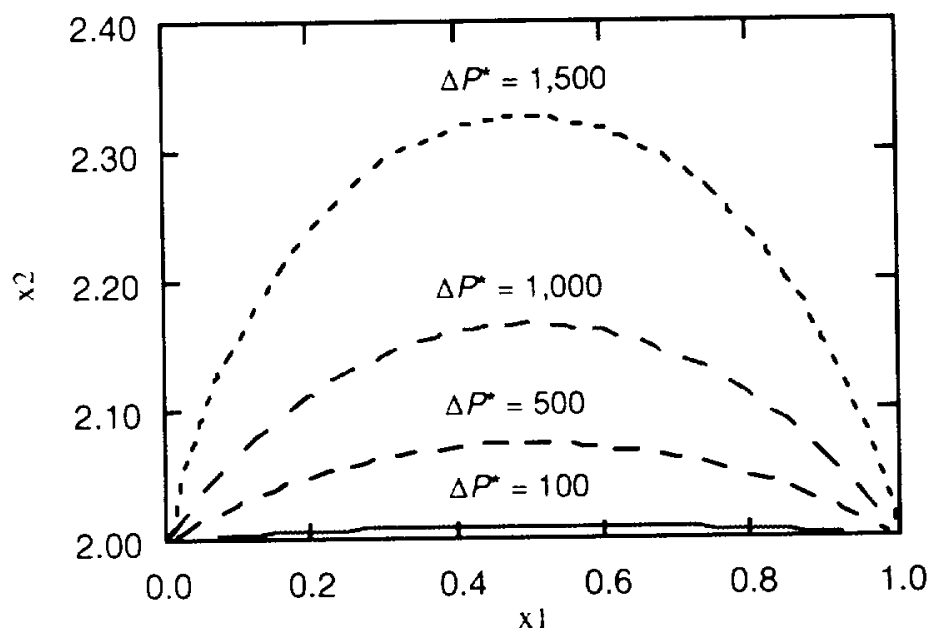

Figure 6. Free surface shape versus pore pressure differential.

\section{Lower Boundary Condition}

As described in section III, the reference model was developed to analyze the parameters associated with the loss of retention in a LAD. In that problem, the lower boundary condition was assumed to be one of uniform flow across the boundary, with the total flow equal to the amount evaporated or condensed. For the case of evaporation, this assumes that the fluid leaving the cavity is being replaced from a large reservoir of constant temperature beneath the interface. For the LAD, a uniform flow boundary condition is valid, however, this boundary condition would not be valid for the porous plug. Physically, a single pore in a porous plug has a large aspect ratio, i.e., the ratio of its length to its diameter, on the order of several hundred. In this study, a pore length of two diameters is used in order to reduce the computation requirements. Therefore, a lower boundary condition which represents a fully developed flow is more appropriate.

To develop the lower velocity boundary condition, the expression for fully developed laminar flow in a channel ${ }^{\text {in }}$ is used and is shown below:

$$
u=u_{\max }\left[1-\left(\frac{x}{a}\right)^{2}\right]
$$


Equation (50) defines a parabolic velocity distribution $u(x)$ that has a value of zero at the sidewalls, $x= \pm a$, and is maximum at the center line, $x=0$. The variable $u_{\max }$ is the maximum value of the velocity distribution, $a$ is the half channel width, and $x$ is the independent variable. Assuming the domain shown in figure 7 , equation (50) can be written as shown in equation (51).

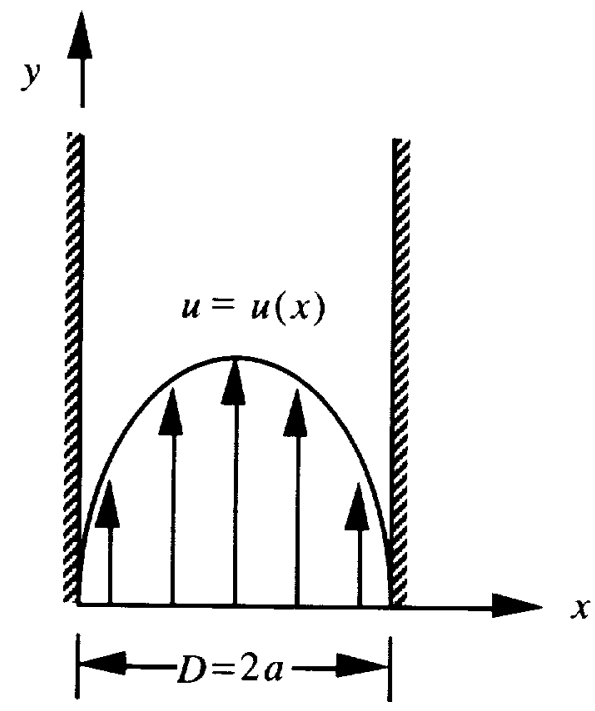

Figure 7. Lower boundary condition coordinate frame.

$$
u(x)=u_{\max }\left[1-\frac{(x-a)^{2}}{a^{2}}\right]
$$
given by:

Using the scaling relations defined in equation (12), equation (51) is nondimensionalized and is

$$
u^{*}=u_{\max }^{*}\left[1-4\left(x^{*}-\frac{1}{2}\right)^{2}\right]
$$
to 1 .

In order to find the total mass flow, $J^{*}$, across the boundary, equation (52) is integrated from 0

$$
J^{*}=\int_{0}^{1} u^{*} d x^{*}=\int_{0}^{1} u_{\max }^{*}\left[1-4\left(x^{*}-\frac{1}{2}\right)^{2}\right] d x^{*}=\frac{2}{3} u_{\max }^{*}
$$

and solving for $u_{\max }$ the following expression is obtained:

where

$$
u_{\max }^{*}=\frac{3}{2} J^{*}
$$

$$
J^{*}=\int_{\Gamma_{4}} j^{*} d \Gamma_{4}
$$


Using equations (52) and (54) along with the total mass flux that is exiting the pore through evaporation, the lower boundary condition is defined. A typical velocity profile is shown in figure 8 .

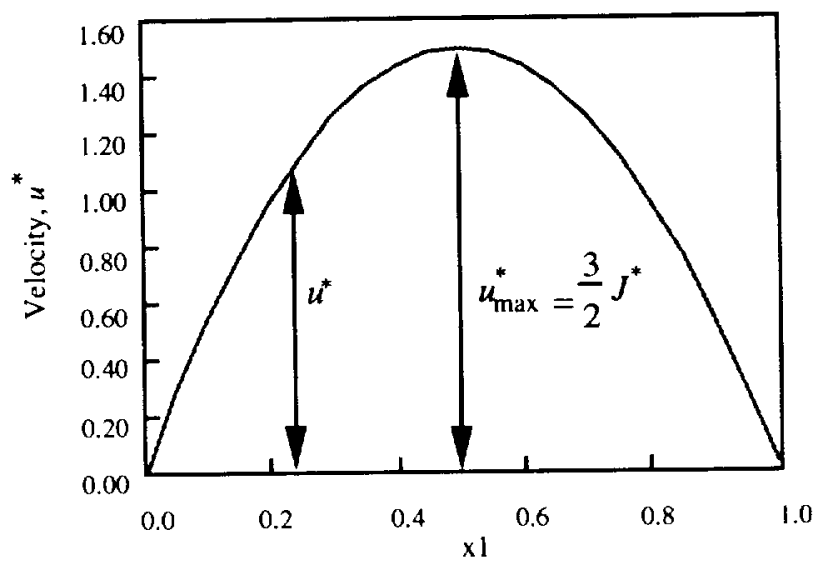

Figure 8. Lower boundary velocity distribution.

\section{Sidewall Boundary Condition}

In the reference model, the sidewall boundary conditions were assumed to be isothermal. The isothermal boundary condition was appropriate for the problem studied ${ }^{8}$ because of the small length scale between the liquid and the vapor; however, for the porous plug, the condition is valid only if the porous plug material is manufactured from a high thermal conductivity material such as copper. If the porous plug is manufactured from a lower thermal conductivity material, the isothermal boundary conditions would not apply, rather there will be a thermal gradient through the material. For this case, a linear temperature gradient will be imposed on the sidewall boundaries of the porous plug, where the magnitude will be based on the plug thermal conductivity.

Figure 9 shows a linear temperature profile along the side boundaries of the porous plug.

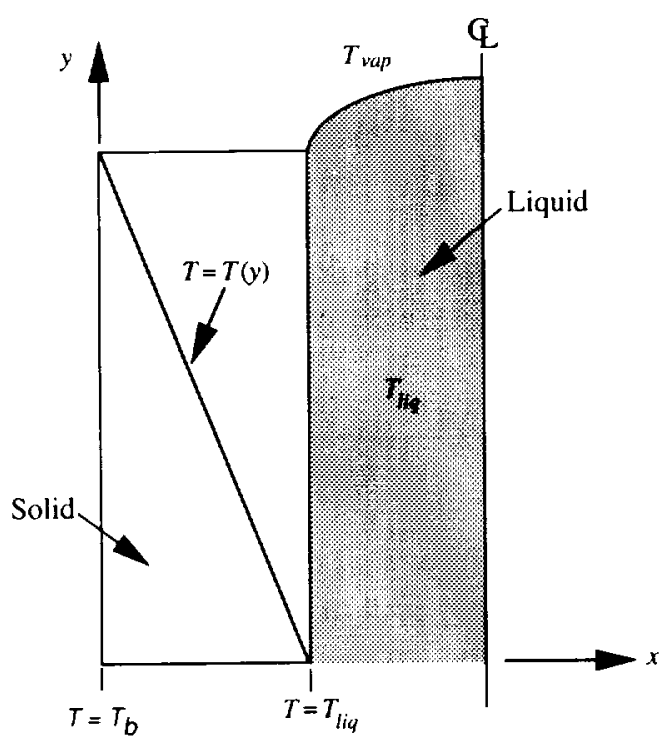

Figure 9. Side boundary temperature distribution. 
In order to model the thermal gradient boundary condition, an assumption that the temperature distribution is linear and can be approximated as a one-dimensional conduction problem is made.

Assuming that the temperature distribution through the solid boundary is linear implies that the thermal conductivity of the material is constant, i.e., the thermal conductivity is independent of temperature. An additional assumption that there is no heat generation from within the plug material is also made. With the preceding assumptions, the equation for the temperature through the plug ${ }^{17}$ is given by:

$$
T_{, y y}=0, \quad @ 0<y<L
$$

with both Dirchlet and Neumann boundary conditions given by:

and

$$
T=T_{\text {liq }}, \quad @ y=0
$$

$$
k T_{, y}+h\left(T-T_{\text {vap }}\right)=0, @ y=L .
$$

Using the appropriate scaling factors given in equation (12), equations (56) through (58) can be nondimensionalized as:

$$
\begin{array}{cl}
T_{, y y}^{*}=0, & @ 0<y^{*}<1 \\
T^{*}=1, & \text { @ } y^{*}=0 \\
T_{, y}^{*}+B i T^{*}=0, & \text { @ } y^{*}=A R
\end{array}
$$

where the aspect ratio, $A R$, is defined as the ratio of the numerical domain length to the pore diameter and where the Biot number, $B i$, is defined as the ratio of the heat transfer by convection to that by conduction across a material of length $L$. A small value of $B i$ implies that the heat transfer is dominated by conduction. Based on this definition, the Biot number takes on the form:

$$
B i=\frac{h L}{k^{(s o l)}}
$$

where $h$ is the convective heat transfer coefficient based on the vapor, $k$ is the thermal conductivity of the plug material, and $L$ is the physical length of the plug.

Integrating equation (59) twice and applying the boundary conditions shown in equations (60) and (61) gives the following relation for the sidewall temperature distribution:

$$
T^{*}=1-\frac{B i}{1+(A R) B i} \times 2
$$

Equation (63) is used as the left and right wall temperature boundary conditions for the case of nonisothermal sidewalls. Figure 10 shows the effect of $B i$ on the temperature distribution through the plug material. 


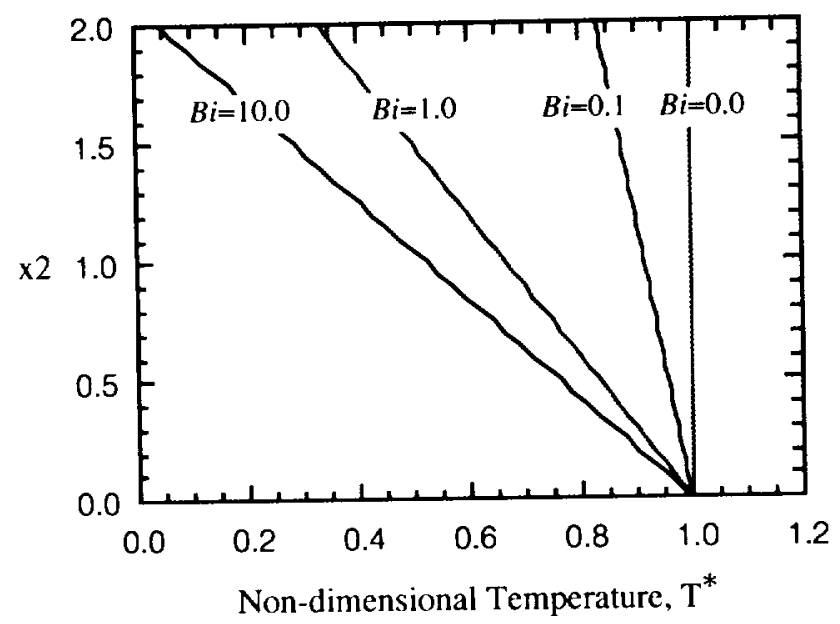

Figure 10. Sidewall temperature for various $B i$.

\section{E. Summary}

In this section, the modifications to the existing model that were required to analyze the porous plug are discussed. The only modifications that were required included some of the boundary conditions, namely, the free surface solution, the redefinition of the bottom velocity boundary condition, and the right and left wall temperature boundary conditions.

\section{RESULTS AND FINDINGS}

\section{A. Introduction}

Using the model discussed in section IV, the porous plug temperature profile, flow field, and mass transfer across the interface are determined for various initial conditions. In this section, the hydrogen properties and associated nondimensional parameters that are used in this study are presented and discussed. Also, a parametric analysis on the model grid size is discussed for the case of combined evaporation/thermocapillary flow. This is done to evaluate the effect of grid refinement on the numerical solution and to choose an appropriate grid resolution for the analysis.

Using the hydrogen parameters identified and the appropriate grid size, three cases are discussed: (1) the basic state, pure evaporation driven flow; (2) combined evaporation/thermocapillary flow; and (3) combined evaporation/thermocapillary flow with a sidewall temperature gradient. Cases (2) and (3) are run for various $\Delta T$ magnitudes across the pore and are compared to case (1).

\section{B. Fluid Properties and Nondimensional Groups}

As was discussed in section II, most prior work in the area of porous plug research has been based on helium as the working fluid. Only cursory examinations have been done using liquid hydrogen. In this section, the relevant hydrogen properties that are used in this study are given in table 1 , and the associated nondimensional groupings are calculated and presented. The hydrogen properties are taken at ambient pressure and saturated conditions. 
Table 1. Saturated hydrogen properties.

\begin{tabular}{|l|l|l|}
\hline \multicolumn{1}{|c|}{ Property } & \multicolumn{1}{c|}{ Units } & \multicolumn{1}{c|}{ Value } \\
\hline$P_{\text {sat }}$ & $(\mathrm{MPa})$ & 0.1013 \\
$T_{\text {sat }}$ & $(\mathrm{K})$ & 20.3 \\
$\rho^{(l)}$ & $\left(\mathrm{kg} / \mathrm{m}^{3}\right)$ & 70.79 \\
$\rho^{(v)}$ & $\left(\mathrm{kg} / \mathrm{m}^{3}\right)$ & 1.339 \\
$\mu^{(l)}$ & $(\mathrm{kg} / \mathrm{m}-\mathrm{s})$ & $1.319 \times 10^{-5}$ \\
$\mu^{(v)}$ & $(\mathrm{kg} / \mathrm{m}-\mathrm{s})$ & $8.5 \times 10^{-7}$ \\
$v^{(l)}$ & $\left(\mathrm{m}^{2} / \mathrm{s}\right)$ & $1.860 \times 10^{-7}$ \\
$v^{(v)}$ & $\left(\mathrm{m}^{2} / \mathrm{s}\right)$ & $6.348 \times 10^{-7}$ \\
$k^{(l)}$ & $(\mathrm{W} / \mathrm{m}-\mathrm{K})$ & $1.182 \times 10^{-1}$ \\
$k^{(v)}$ & $(\mathrm{W} / \mathrm{m}-\mathrm{K})$ & $1.883 \times 10^{-2}$ \\
$C p^{(l)}$ & $(\mathrm{J} / \mathrm{kg}-\mathrm{K})$ & $9.689 \times 10^{3}$ \\
$C p^{(v)}$ & $(\mathrm{J} / \mathrm{kg}-\mathrm{K})$ & $1.176 \times 10^{4}$ \\
$\alpha^{(l)}$ & $\left(\mathrm{m}^{2} / \mathrm{s}\right)$ & $1.440 \times 10^{-7}$ \\
$\alpha^{(v)}$ & $\left(\mathrm{m}^{2} / \mathrm{s}\right)$ & $1.042 \times 10^{-6}$ \\
$L$ & $(\mathrm{~J} / \mathrm{kg})$ & $4.456 \times 10^{5}$ \\
$M_{w}$ & $(\mathrm{~kg} / \mathrm{kgmol})$ & 2.016 \\
$\gamma$ & $(\mathrm{N} / \mathrm{m})$ & $1.973 \times 10^{-3}$ \\
$|d \gamma / d T|$ & $(\mathrm{N} / \mathrm{m}-\mathrm{K})$ & $1.620 \times 10^{-4}$ \\
$\beta$ & $(1 / \mathrm{K})$ & $1.569 \times 10^{-2}$ \\
$R_{g}$ & $(\mathrm{~J} / \mathrm{kmol}-\mathrm{K})$ & $8.314 \times 10^{3}$ \\
\hline
\end{tabular}

Using the property values given in table 1, the corresponding relevant nondimensional groups that are independent of the temperature differential, $\Delta T$, across the pore are given in table 2 . For all nondimensional parameters, a pore diameter of $1 \mu \mathrm{m}$ and a coefficient of evaporation of 0.5 are used.

Table 2. Nondimensional groups.

\begin{tabular}{|c|l|}
\hline Parameter & Value \\
\hline$N u$ & $7.639 \times 10^{1}$ \\
$B o$ & $3.52 \times 10^{-7}$ \\
$C a$ & $1.241 \times 10^{-3}$ \\
$f_{\rho}$ & $5.287 \times 10^{1}$ \\
$\lambda$ & $1.911 \times 10^{-1}$ \\
$\Lambda$ & $1.288 \times 10^{7}$ \\
$P r$ & 1.292 \\
\hline
\end{tabular}


Based on the value of $C a$ given in table 2, the nondimensional bubble point, $\Delta P^{*}{ }_{b}=2 / C a$, is equal to 1,611 .

Table 3 gives the relevant nondimensional groups that are dependent on $\Delta T$. Also, for the case where $M a$ is nonzero, $C a$ can be defined as $C r P r / M a$ and the nondimensional bubble point, $\Delta P^{*}{ }_{b}=$ $2 \mathrm{Ma} / \mathrm{CrPr}$, is equal to 1,608 . In this study, the effect of $\Delta P$ across the pore is not examined, but is set at a fixed value of 1,000 .

Table 3. Nondimensional groups versus $\Delta T$.

\begin{tabular}{|c|c|c|c|}
\hline Parameter & $\Delta T=1.0$ & $\Delta T=1.25$ & $\Delta T=1.5$ \\
\hline$C r$ & $8.211 \times 10^{-2}$ & $1.026 \times 10^{-1}$ & $1.232 \times 10^{-1}$ \\
$E$ & $2.015 \times 10^{-2}$ & $2.518 \times 10^{-2}$ & $3.022 \times 10^{-2}$ \\
$G r$ & $4.449 \times 10^{-6}$ & $5.561 \times 10^{-6}$ & $6.674 \times 10^{-6}$ \\
$M a$ & $8.529 \times 10^{1}$ & $1.066 \times 10^{2}$ & $1.279 \times 10^{2}$ \\
$R s$ & $6.498 \times 10^{-1}$ & $5.199 \times 10^{-1}$ & $4.332 \times 10^{-1}$ \\
$V r$ & $9.908 \times 10^{-2}$ & $1.238 \times 10^{-1}$ & $1.486 \times 10^{-1}$ \\
\hline
\end{tabular}

\section{Grid Refinement}

In order to determine the appropriate grid size to use in this study, the case of combined evaporation/thermocapillary flow is used. For this grid refinement exercise, the values of the nondimensional groups used are given in tables 2 and 3 for $\Delta T=1$. For this case, four grid sizes were chosen where the number of elements in the $x 1$ direction is given by $N X E$, and the number of elements in the $x 2$ direction is given by $N Y E$. Grid sizes of $10 \times 15 \rightarrow 25 \times 30$ in increments of five elements for $N X E$ and $N Y E$ are used, where the total number of grid points is equal to $(2 * N X E+1) *(2 * N Y E+1)$. The grid size of $10 \times 15$ corre sponded to the coarsest grid required for adequate velocity convergence, and the grid size of $25 \times 30$ corresponded to the finest grid that could be solved using the available computer resources.

To evaluate the effect of grid size, the surface temperature distributions, the mean surface temperature, and the evaporation flux for the four grid sizes are compared. The surface temperature is used since it drives the entire flow field, either by causing evaporation across the interface or by causing thermocapillary convection on the surface.

For the case of combined flow with a $\Delta T=1$, the surface temperature gradient for various grid resolutions is given in figure 11 .

The magnitude of the gradient and the percent change due to grid refinement is shown in table 4 .

In addition to the surface temperature distribution, the evaporation flux is also used to evaluate the grid size. Table 5 gives the percent change in $J^{*}$ due to grid refinement. 


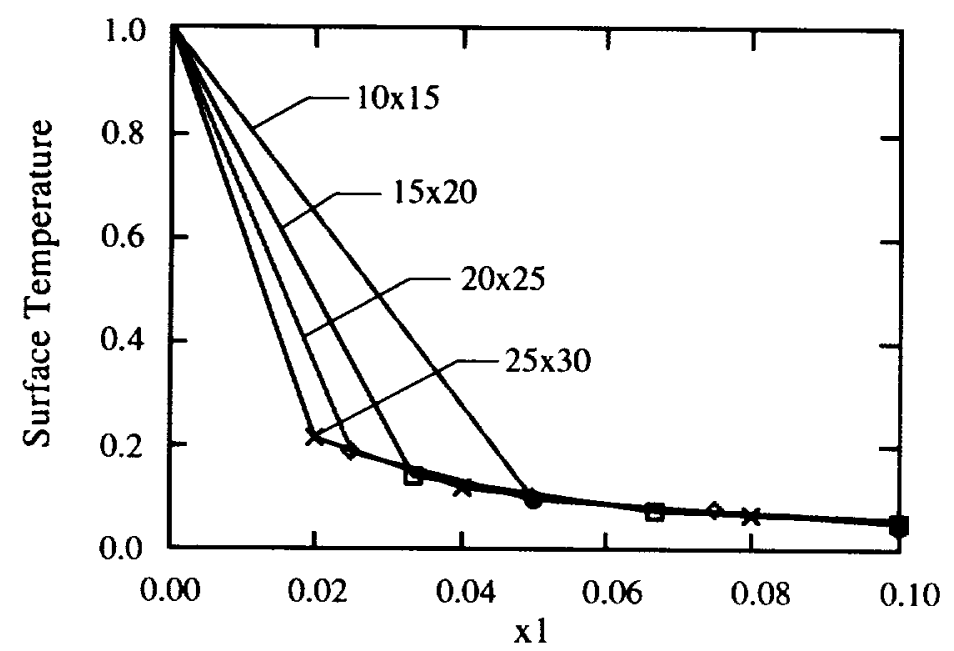

Figure 11. Surface temperature for various grid sizes, $\Delta T=1$.

Table 4. Surface temperature gradient sensitivity to grid size, $\Delta T=1$.

\begin{tabular}{|c|c|c|}
\hline Grid Size & $T_{x}$ & $\begin{array}{c}\text { Percent } \\
\text { Change }\end{array}$ \\
\hline $10 \times 15$ & -17.98 & - \\
$15 \times 20$ & -25.67 & 42.8 \\
$20 \times 25$ & -32.62 & 27.1 \\
$25 \times 30$ & -39.05 & 19.7 \\
\hline
\end{tabular}

Table 5. Evaporation flux sensitivity to grid size, $\Delta T=1$.

\begin{tabular}{|c|c|c|}
\hline Grid Size & $J^{*}$ & $\begin{array}{c}\text { Percent } \\
\text { Change }\end{array}$ \\
\hline $10 \times 15$ & 0.077 & - \\
$15 \times 20$ & 0.082 & 6.5 \\
$20 \times 25$ & 0.086 & 4.9 \\
$25 \times 30$ & 0.089 & 3.5 \\
\hline
\end{tabular}

As is shown in both table 4 and 5 , the increased number of elements or grid points serves to help resolve the large temperature gradient in the pore corners and in the overall evaporation flux across the pore.

Although a grid size of $25 \times 30$ gives approximately a 20-percent change in $T_{x}$ from the previous grid, the value of $J^{*}$ changes by only 3.5 percent. Therefore, due to the relatively small change in $J^{*}$ and due to the exorbitant computational requirements of the $25 \times 30$ grid, a grid size of $20 \times 25$ is used in this study. It should be noted that a more refined grid should help to resolve the corner temperature gradient further and produced more accurate results in $J^{*}$. 


\section{Basic State (Pure Evaporation)}

For the purpose of this study, the basic state for the porous plug analysis will be defined as pure evaporation across the interface, i.e., setting the Marangoni number equal to zero with a $\Delta T=1.0 \mathrm{~K}$. All other nondimensional parameters are as shown in tables 2 and 3 for $\Delta T=1.0 \mathrm{~K}$ are used. Also, the sidewall temperature boundary condition is taken as isothermal having a value of 1.0 .

Figure 12 shows the temperature and stream function profile that results due to the latent heat transport across the surface due to evaporation. The stream function profile indicates that the liquid is being driven toward the corners of the pore due to the increased evaporation at the regions of higher surface temperature.
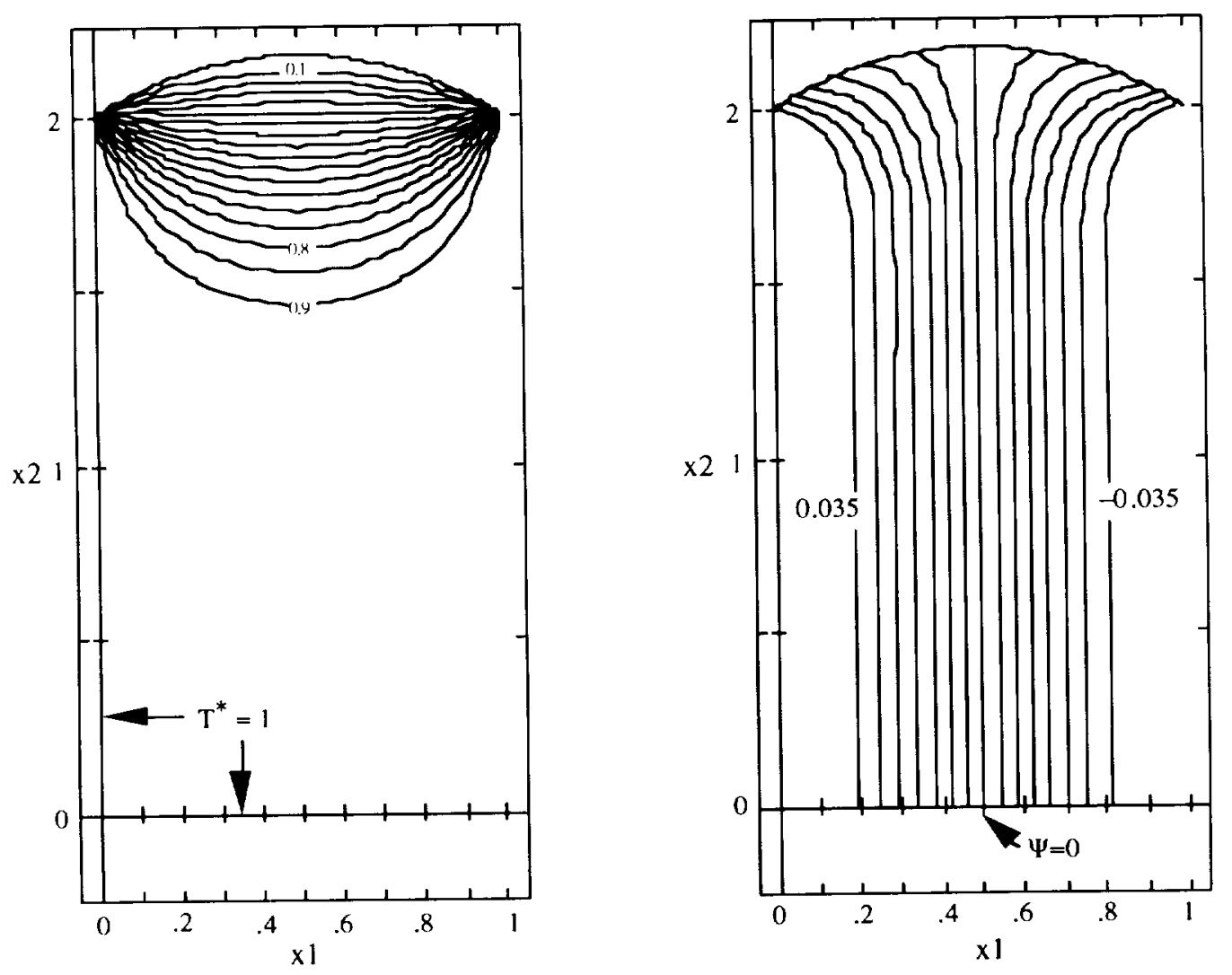

Figure 12. Basic state temperature and stream function distribution.

Figure 13 shows the surface temperature distribution for the basic state. As shown, there is a large surface temperature gradient in the corner regions of the pore.

Based on this surface temperature distribution, the mean surface temperature, $\bar{T}_{\text {surf }}$, is equal to 0.088 , or in dimensional form $19.38 \mathrm{~K}$, and total mass transfer or evaporation, $J^{*}$, across the surface is calculated using equation (24) and is found to be equal to 0.086 , or in dimensional form, $1.136 \mathrm{~kg} / \mathrm{m}^{2}-\mathrm{s}$. 


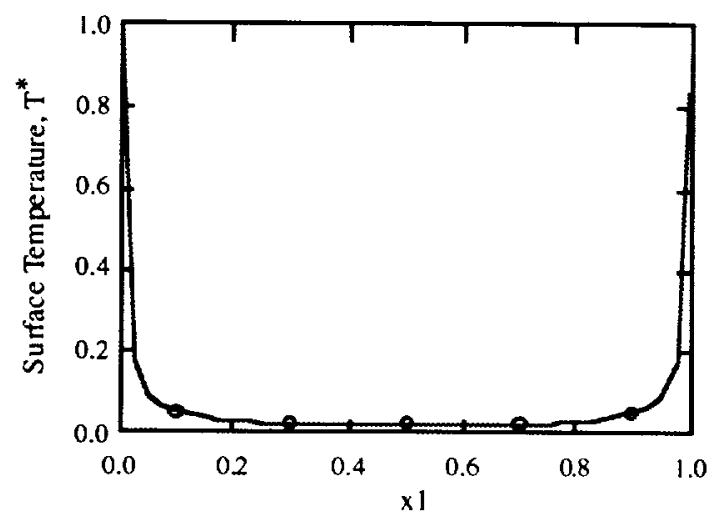

Figure 13. Basic state surface temperature distribution.

\section{E. Combined Evaporation/Thermocapillary Flow}

The case of combined flow is indicative of an actual pore due to the presence of thermocapillary convection. This section assumes combined flow while specifying isothermal sidewall boundary conditions, i.e., $T_{\text {wall }}=1.0$. As can be seen in figure 14 , no significant change in the isotherms is observed, but considerable change in the flow field is apparent. The flow field for combined flow is characterized by two cells of equal and opposite stream function distributions. This flow field results from the liquid on the surface being driven into the center of the pore due to the variation of surface tension. Figure 14 shows the temperature and stream function distribution for combined flow with an imposed $\Delta T=1.0 \mathrm{~K}$.
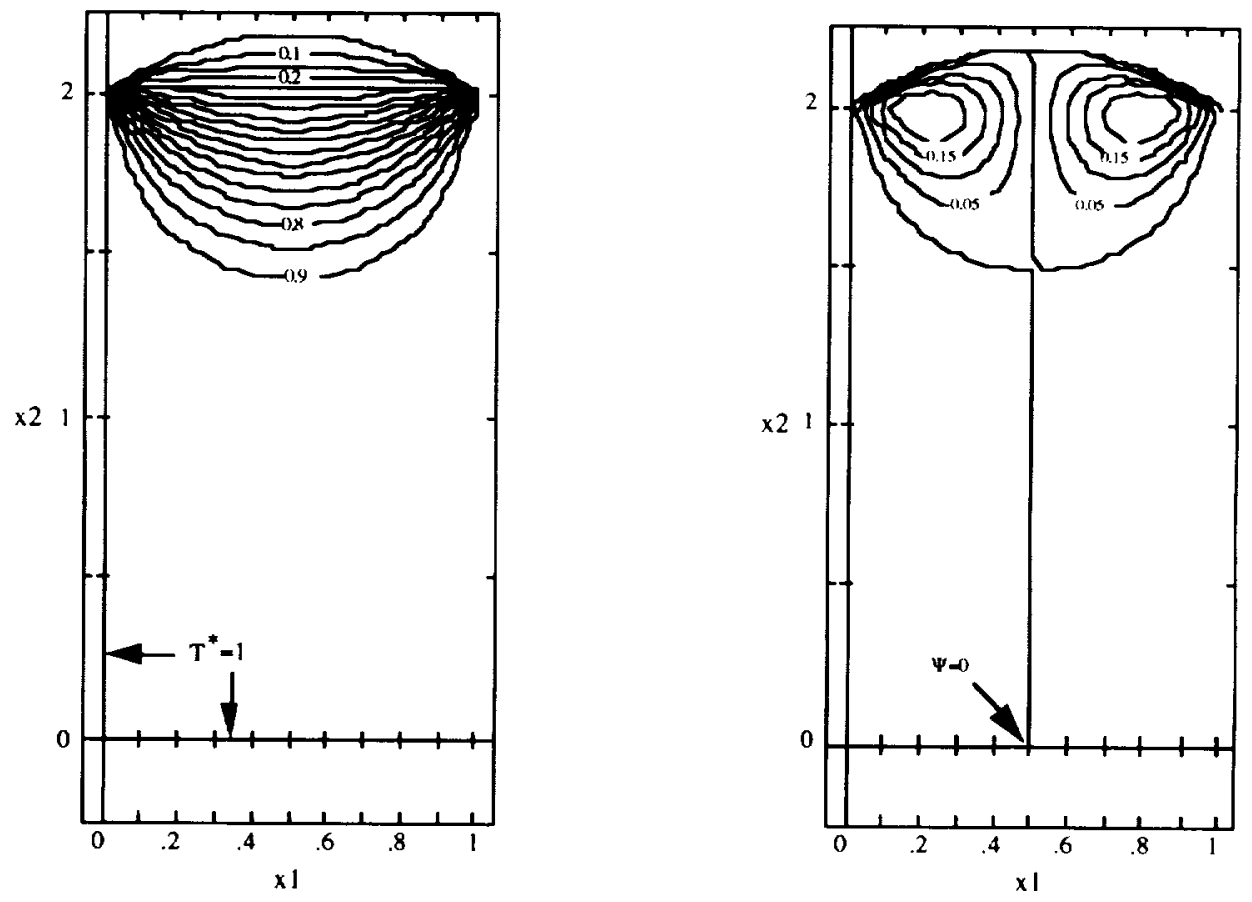

Figure 14. Temperature and stream function distribution, $M a=85.29$. 
For increasing levels of superheat, i.e., $\Delta T$, Marangoni number will also increase due to its dependence on $\Delta T$, thus increasing the liquid convection from the hot boundary, namely the sidewalls, to the center of the cavity. This liquid convection from the sidewall to the center will tend to increase $\bar{T}_{\text {surf }}$ by transporting warmer liquid along the surface toward the center of the pore. However, this increase in surface temperature will result in an increase in the evaporation across the interface, which tends to decrease $\bar{T}_{\text {surf }}$ due to the latent heat transport from the liquid to the vapor. In addition, the increased evaporation will tend to drive the liquid to the corners, as evidenced in figure 12 . Table 6 gives the values for $\bar{T}_{\text {surf }}$ and $J^{*}$ for various levels of super heat.

Table 6. $\bar{T}_{\text {surf }}$ and $J^{*}$ versus $\Delta T$.

\begin{tabular}{|l|c|c|}
\hline$\Delta T(\mathrm{~K})$ & $\bar{T}_{\text {surf }}$ & $J^{*}$ \\
\hline Basic & 0.088 & 0.086 \\
1.0 & 0.087 & 0.086 \\
1.25 & 0.086 & 0.107 \\
1.5 & 0.086 & 0.129 \\
\hline
\end{tabular}

Using the scaling relations given in equation (12) and the relevant properties given in table 1 , the dimensional values of $\bar{T}_{\text {surf }}$ and $J$ are given in table 7 .

Table 7. $\bar{T}_{\text {surf }}$ and $J^{*}$ versus $\Delta T$ (dimensional).

\begin{tabular}{|l|c|c|}
\hline$\Delta T(\mathrm{~K})$ & $\bar{T}_{\text {surf }}(\mathrm{K})$ & $J\left(\mathrm{~kg} / \mathrm{m}^{2}-\mathrm{s}\right)$ \\
\hline Basic & 19.38 & 1.136 \\
1.0 & 19.38 & 1.132 \\
1.25 & 19.16 & 1.410 \\
1.5 & 18.93 & 1.699 \\
\hline
\end{tabular}

Figure 15 show the variation of the pore mean surface temperature, $\bar{T}_{\text {surf }}$, and evaporation flux, $J^{*}$, respectively.
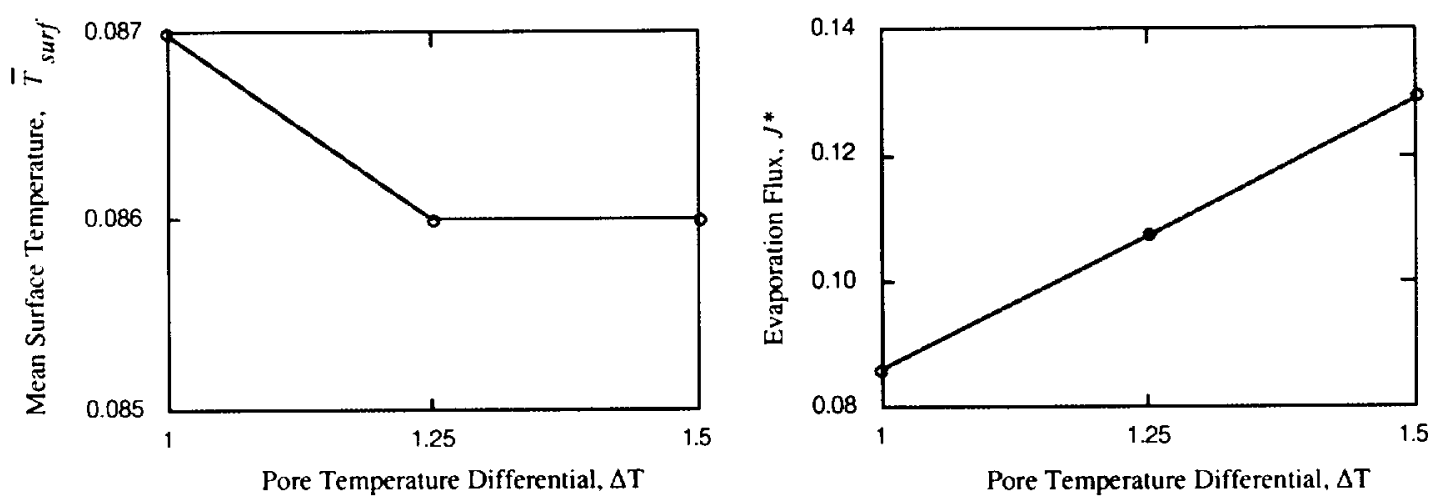

Figure 15. $\bar{T}_{\text {surf }}$ and $J^{*}$ versus $\Delta T$. 
Although $\bar{T}_{\text {surf }}$ is nonlinear over the range of $\Delta T$ considered, $J^{*}$ is linear since it depends on the actual surface temperature, $T^{*}$, and little variation of the actual surface temperature is observed. It was shown by Schmidt ${ }^{8}$ that surface temperature is strongly dependent on the Nusselt number, $N u$, which is independent of $\Delta T$. It was also shown that large values of $M a$ would influence the temperature distribution by convecting the warm liquid toward the center, whereas at lower values of $M a$, similar to the values used in this study, no significant effect on temperature was observed. Therefore, $J^{*}$ is inversely proportional to $R s$, i.e., $J^{*} \propto 1 / R s$ where $R s$ decreases with increasing $\Delta T$.

Another effect of combined flow is the influence of the dynamic pressure on the surface curvature. For the case of evaporation, the liquid is superheated with respect to the vapor and the thermocapillary stress causing the liquid to be driven out of the corner regions, thus causing a pressure decrease in this region. Conservation of mass causes the center portion of the surface to rise above that of the basic state. Figure 16 shows the divergence of the surface position from the basic state with increasing $\Delta T$.

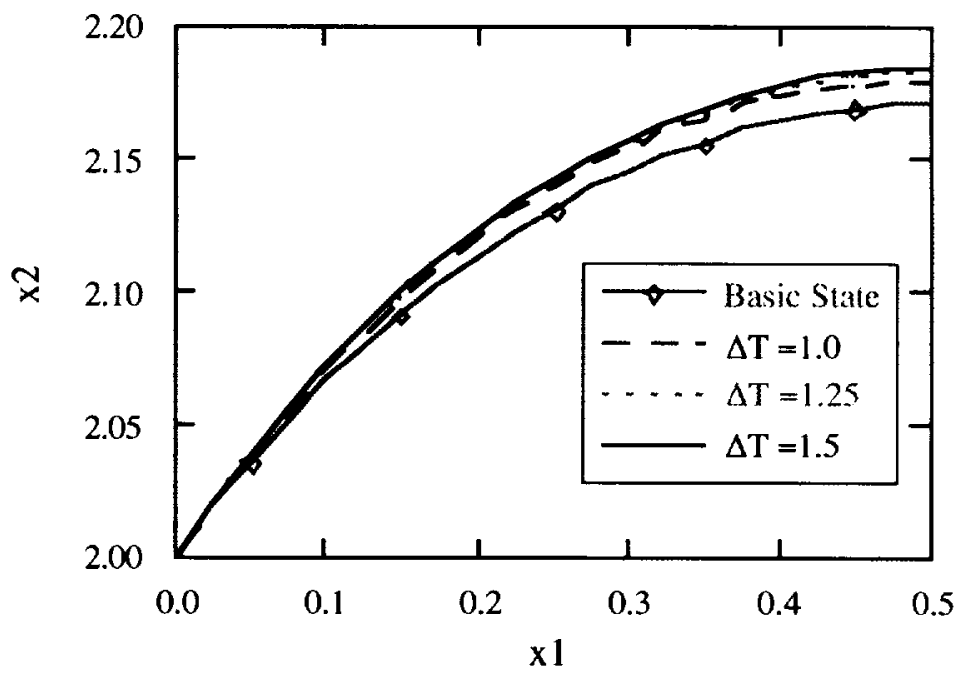

Figure 16. Surface divergence from basic state for various $\Delta T$.

\section{F. Combined Flow With Sidewall Temperature Gradient}

In order to evaluate the effect of a low thermal conductivity porous plug material, the sidewall boundary condition is adjusted to account for a temperature gradient through the porous plug. The magnitude of the gradient is determined by the Biot number, $B i$, specified at the porous plugs upper solid boundary. The Biot number is defined as the ratio of convective heat transfer from the solid/vapor to conductive heat transfer from the liquid/solid. A low value of $B i$ specifies that the heat transfer is dominated by conduction, which is indicative of materials which have high thermal conductivities such as copper. Three values of $B i$ are used to evaluate this effect, $B i$ equal to $0.0,0.15$, and 0.5 , and the corresponding sidewall temperature distributions are shown in figure 17.

Using the above stated values of $B i$, the resultant temperature and flow field is determined and the evaporative mass transfer calculated. Figure 18 shows a typical temperature and flow field for a porous plug with an imposed temperature gradient on the sidewall boundaries. 


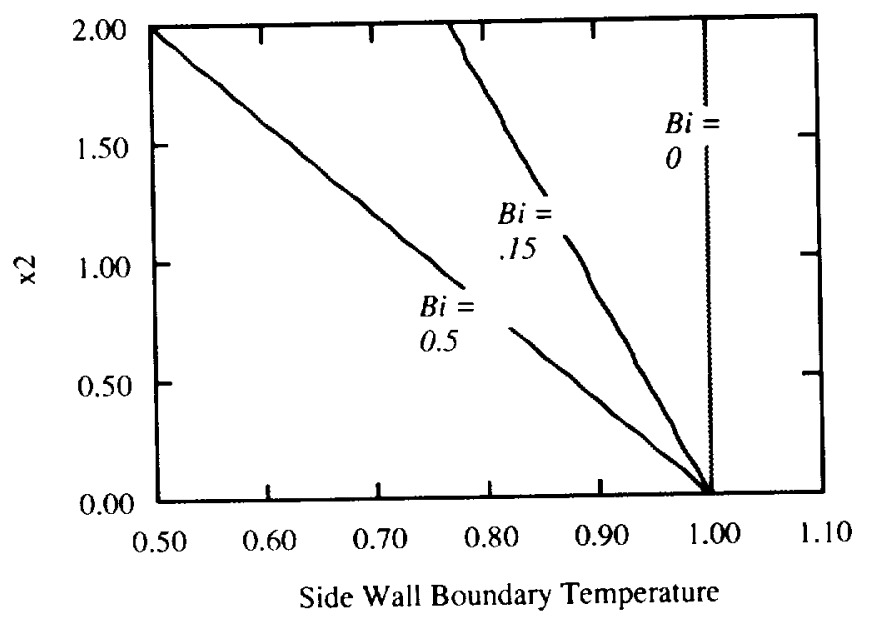

Figure 17. Sidewall boundary temperature for various $B i$.
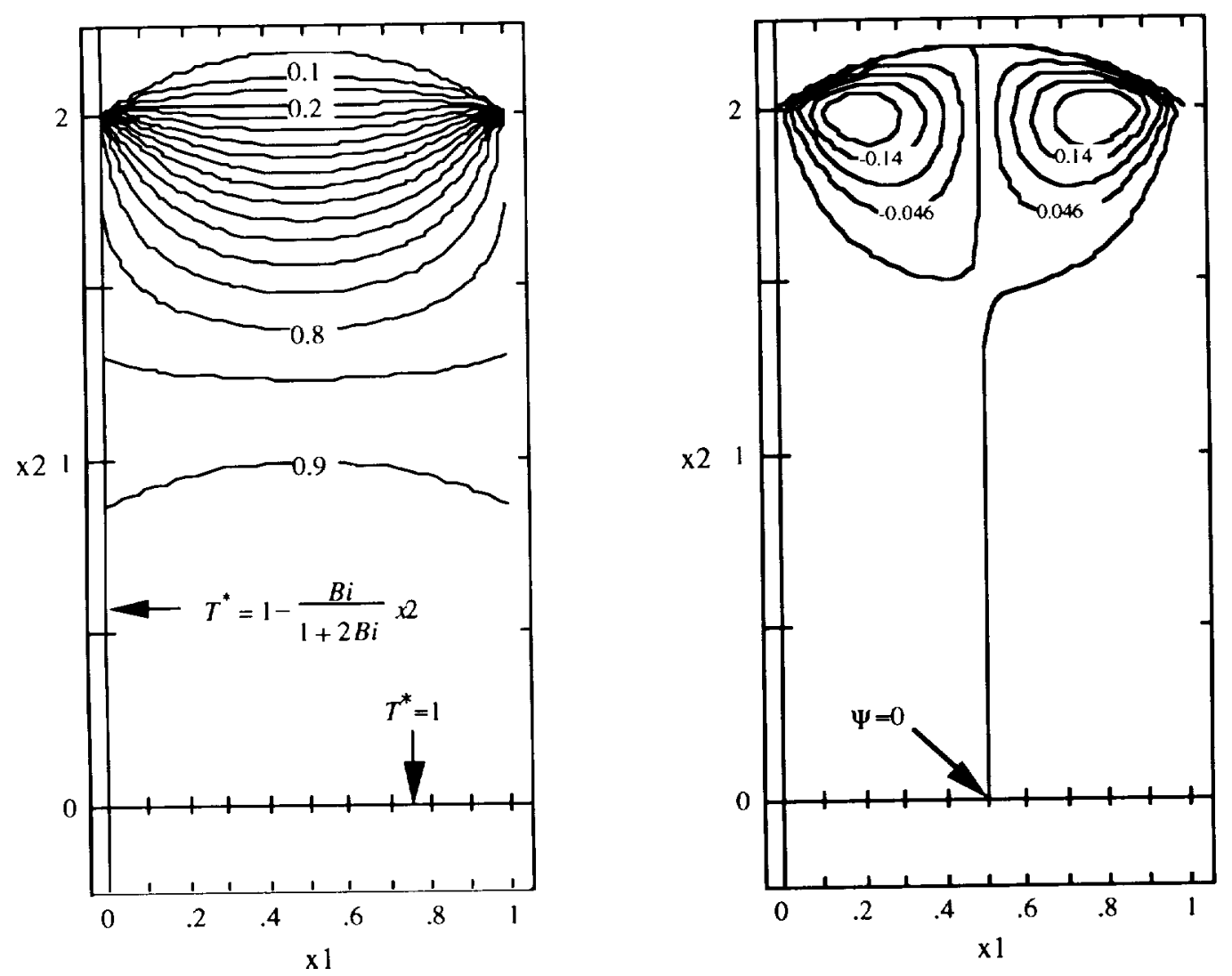

Figure 18. Temperature and stream function distribution, $M a=85.29, B i=0.15$.

The effect of an imposed sidewall temperature gradient is most evident in the solution for the surface temperature distribution. Figure 19 shows the effect of $B i$ on the resultant surface temperature for $\Delta T=1$. As $B i$ is increased, the heat transfer through the plug solid material is becoming dominated by convection on the vapor side, thus the surface remains cooler. This lowering of the surface temperature results in a decrease in the evaporation across the interface as shown in tables 8 through 11. 


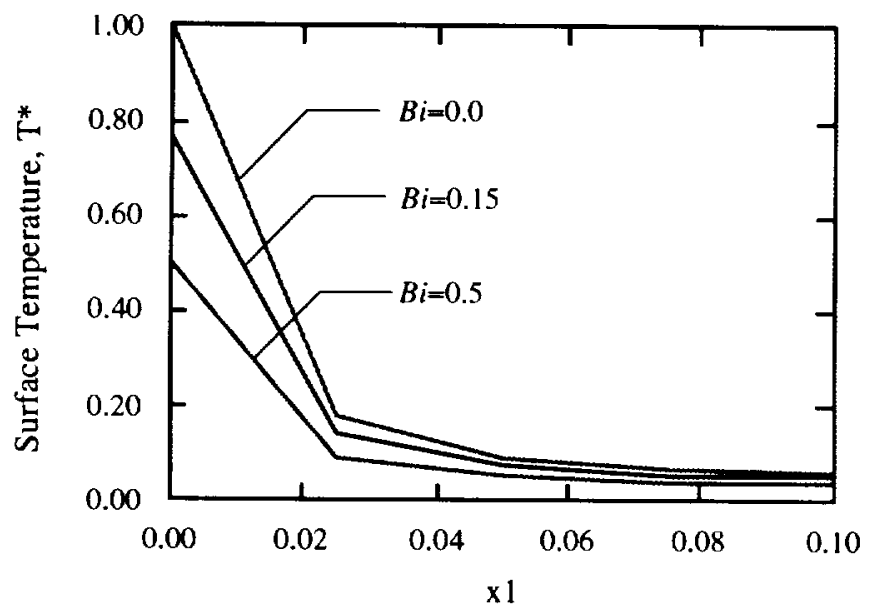

Figure 19. Surface temperature for various $B i, \Delta T=1.0$.

As shown in table $8, \bar{T}_{\text {surf }}$ shows the same variation with $\Delta T$ as was observed in table 6 , with increasing $B i$ causing a lowering of the curve or a decrease in $\bar{T}_{\text {surf }}$.

Table 8. $\bar{T}_{\text {surf }}$ versus $B i$ and $\Delta T$.

\begin{tabular}{|c|c|c|c|}
\hline $\begin{array}{c}\text { Biot No. } \\
(B i)\end{array}$ & $\Delta T=1.0$ & $\begin{array}{c}\bar{T}_{\text {surf }} \\
\Delta T=1.25\end{array}$ & $\Delta T=1.5$ \\
\hline Basic & 0.088 & - & - \\
0.00 & 0.087 & 0.086 & 0.086 \\
0.15 & 0.068 & 0.068 & 0.068 \\
0.50 & 0.046 & 0.046 & 0.046 \\
\hline
\end{tabular}

Using the scaling relations given in equation (12) and the relevant properties given in table 1 , the dimensional values of $\bar{T}_{\text {surf }}$ are given in table 9 .

Table 9. $\bar{T}_{\text {surf }}$ versus $B i$ and $\Delta T$ (dimensional).

\begin{tabular}{|c|c|c|c|}
\hline $\begin{array}{c}\text { Biot No. } \\
(B i)\end{array}$ & $\Delta T=1.0$ & $\begin{array}{c}\bar{T}_{\text {surf }}(\mathrm{K}) \\
\Delta T=1.25\end{array}$ & $\Delta T=1.5$ \\
\hline Basic & 19.38 & - & - \\
0.00 & 19.38 & 19.15 & 18.93 \\
0.15 & 19.36 & 19.13 & 18.90 \\
0.50 & 19.34 & 19.10 & 18.87 \\
\hline
\end{tabular}

Based on the surface temperature distribution given in tables 8 and 9 , the resultant evaporation for the range of superheats considered are given in tables 10 and 11 . 
Table 10. $J^{*}$ versus $B i$ and $\Delta T$.

\begin{tabular}{|c|c|c|c|}
\hline $\begin{array}{c}\text { Biot No. } \\
(\mathrm{Bi})\end{array}$ & $\Delta T=1.0$ & $\Delta T=1.25$ & $\Delta T=1.5$ \\
\hline Basic & 0.086 & - & - \\
0.00 & 0.086 & 0.107 & 0.129 \\
0.15 & 0.068 & 0.086 & 0.103 \\
0.50 & 0.047 & 0.061 & 0.073 \\
\hline
\end{tabular}

Using the scaling relations given in equation (12) and the relevant properties given in table 1 , the dimensional values of $J$ are given in table 11 .

Table 11. $J^{*}$ versus $B i$ and $\Delta T$ (dimensional).

\begin{tabular}{|c|c|c|c|}
\hline $\begin{array}{c}\text { Biot No. } \\
(B i)\end{array}$ & $\Delta T=1.0$ & $\begin{array}{c}J\left(\mathrm{~kg} / \mathrm{m}^{2}-\mathrm{s}\right) \\
\Delta T=1.25\end{array}$ & $\Delta T=1.5$ \\
\hline Basic & 1.136 & - & - \\
0.00 & 1.132 & 1.410 & 1.699 \\
0.15 & 0.905 & 1.132 & 1.357 \\
0.50 & 0.629 & 0.804 & 0.969 \\
\hline
\end{tabular}

Figure 20 shows the same linear trend of $J^{*}$ versus $\Delta T$ across the pore as was shown in figure 15 . The effect $B i$ is to lower the curve, while keeping the same general slope.

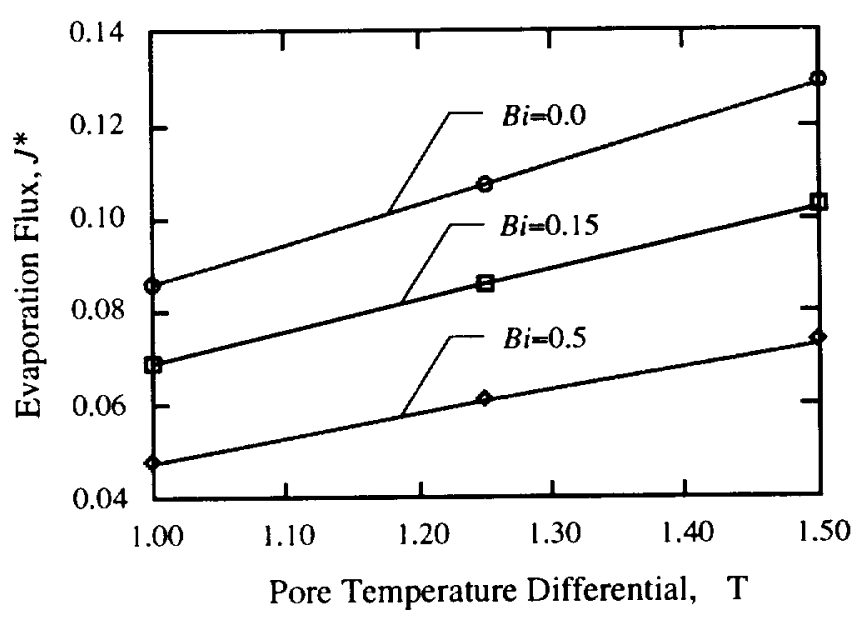

Figure 20. $J^{*}$ versus $\Delta T$ for various $B i$.

Figure 21 shows that the variation of evaporation flux, $J^{*}$ as a function of $B i$ is nonlinear and decreases as $B i$ is increased. 


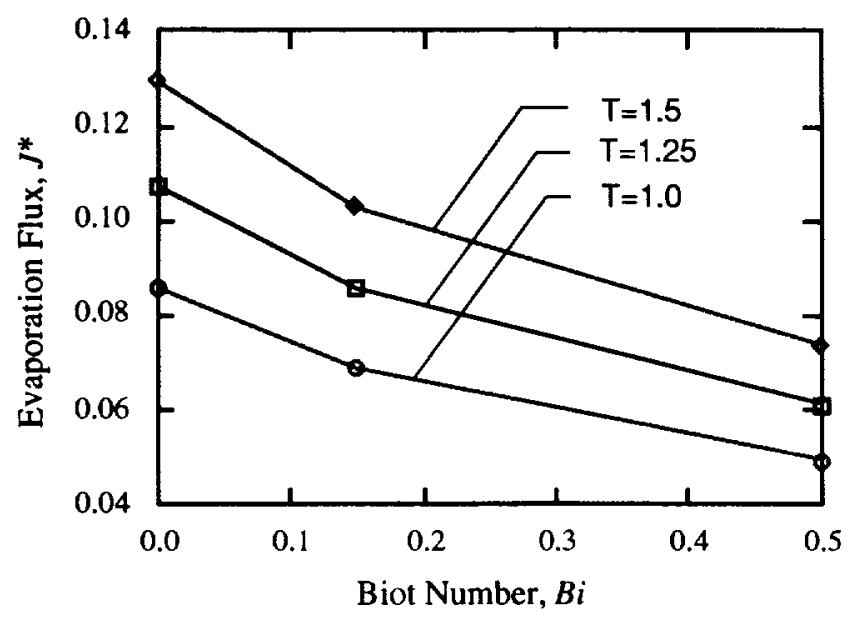

Figure 21. $J^{*}$ versus $B i$ for various $\Delta T$.

\section{G. Summary}

In this section, the relevant fluid properties and nondimensional groups for liquid hydrogen are presented. Superheats of $1.0,1.25$, and $1.5 \mathrm{~K}$ are used in defining the nondimensional groups that are dependent on $\Delta T$. To evaluate the effect of grid refinement on the solution, various grid sizes were examined. The parameters used to evaluate this effect are the corner surface temperature gradient, $T_{, x}$, and the resultant evaporation flux across the pore. Grid sizes of $10 \times 15,15 \times 20,20 \times 25$, and $25 \times 30$ were used. It was found that as the grid is refined, i.e., the number of grid points is increased, both $T,{ }_{x}$ and $J^{*}$ are converging on their exact value. Although a grid size of $25 \times 30$ gave the smallest percent change from the pervious grid, a grid size of $20 \times 25$ is used in this study due to computer limitations.

The steady state temperature distribution and flow field for the case of pure evaporation was solved and used as the basic state for comparison with the combined flow and sidewall thermal gradient boundary condition. The basic state was also compared to pure thermal conduction in the liquid. It was observed that the mean surface temperature, $\bar{T}_{\text {surf }}$, decreased with evaporation due to the transfer of the latent heat across the interface.

The steady-state temperature distribution and flow field for the case of combined evaporation/ thermocapillary flow was solved for various levels of liquid superheat. Pore temperature differentials, $\Delta T$, of $1.0,1.25$, and $1.5 \mathrm{~K}$ were used. It was shown that as the total evaporation across the pore, $J^{*}$, is directly proportional with $\Delta T$.

The steady-state temperature distribution and flow field for the case of combined evaporation/ thermocapillary flow with an imposed sidewall thermal gradient was solved using various values for the Biot number, $B i$, i.e., the magnitude of convection from the pore solid boundary to the magnitude of solid conduction through the pore. Biot numbers of $0.0,0.15$, and 0.5 were used over the same range of $\Delta T$ used previously. As with the case of combined evaporation/thermocapillary flow with isothermal boundaries, $J^{*}$ is directly proportional and linear with $\Delta T$. The effect of $B i$ is to shift the curve down, i.e., as $B i$ increases the total evaporation across the pore decreases. This decrease in $J^{*}$ is inversely proportional with $B i$ and nonlinear. 


\section{DISCUSSION AND CONCLUSIONS}

\section{A. Introduction}

The objective of this study was to investigate some of the physical parameters that influence the mass transport within a porous plug trapping device. In this study, the influence of parameters such as the level of superheat and the magnitude of an imposed thermal gradient along the sidewall was shown. The influence of other parameters such as $\mathrm{Ma}, \mathrm{Cr}, \mathrm{Rs}, \mathrm{Gr}$, and $\mathrm{Vr}$ were also indirectly investigated since all depend on the liquid superheat, i.e., $\Delta T$ across the pore.

The effect of these parameters on the thermal distribution, flow field, and total evaporation is discussed.

\section{B. Discussion}

1. Basic State (Pure Evaporation). The basic state as defined in this study is the temperature distribution, flow field, and total evaporation in a pore having a diameter of $1 \mu \mathrm{m}$, a liquid superheat of $1 \mathrm{~K}$, and a vanishing Marangoni number. The flow field resulting from the basic state conditions is vertically oriented, i.e., the velocity vectors are directed from the bottom of the pore to the top and exit the pore through evaporation across the free surface. The only effect that drives the flow field in this case is the mass transport across the interface. Near the interface, the flow is directed toward the corners of the pore due to the high surface thermal gradient that occurs in the corners. Due to the high thermal gradient in the corner regions, the majority of the evaporation occurs in the corner areas with very little evaporation at the pore center.

Liquid convection due to the mass transport across the interface provides surface cooling by driving the cooler liquid in the center of the pore to the warm corner regions and by latent heat transport at the liquid surface.

2. Combined Evaporation/Thermocapillary Flow. The flow field which results from the combined evaporation/thermocapillary driven flow consists of two counter-rotating cells near the liquid surface. The cells are formed by liquid convection along the surface caused by the variation of surface tension. For this study, the surface tension is defined as being a decreasing linear function of temperature. Since the surface temperature decreases toward the center of the cavity, the liquid is convected toward the center.

Thermocapillary convection will tend to raise the surface temperature by convecting warmer liquid from the pore corner regions to the cooler center, i.e., opposes the previously described evaporation effects where the cooler liquid in the center of the pore is convected toward the corners. As was shown in tables 6 and 7 , as the $\Delta T$ across the pore increased, the liquid evaporation also increased while the mean surface temperature decreased. This would seem to indicate that the convection due to the evaporation and latent heat transport seemed to dominate the convection arising from thermocapillarity. Although, it was shown by $\mathrm{Schmidt}^{8}$ that for very large values of $M a$, i.e., $M a>1,000, \bar{T}_{\text {surf }}$ will tend to increase.

3. Combined Flow With Sidewall Temperature Gradient. The flow field that results from combined flow with sidewall temperature gradient consists of two counter-rotating cells much like the flow field for isothermal sidewalls. The most significant difference relative to combined 
evaporation/thermocapillary driven flow is the steady-state thermal distribution. The effect of a sidewall $B i$ is the formation of a thermal gradient throughout the body of the liquid. This is in contrast to the results discussed previously where the thermal gradient was restricted to the region about the free surface. Again, liquid is convected along the free surface and drives the warmer liquid from the corner regions to the cooler pore center.

The most significant effect of $B i$ is the lowering of the overall surface temperature distribution, which is evident in both the actual temperature distribution across the pore and in $\bar{T}_{\text {surf }}$. The surface temperature and temperature gradient in the corner regions decreases with increasing $B i$. This reduction in temperature occurs since the heat conduction from the pore interior to the surface is reduced; thus, the sidewall temperature is lower than if the wall were isothermal, $T_{1,2}=1.0$. Since less heat is transported to the free surface, the overall evaporation across the interface is reduced accordingly. The variation of

$J^{*}$ with $\Delta T$ is linear with the effect of $B i$ causing the curve to be lowered with increasing $B i$. A secondary observation is that the variation of $J^{*}$ with $B i$ is nonlinear and decreasing. In this case, the effect of $\Delta T$ is to raise the curve with increasing $\Delta T$.

\section{Conclusions}

The results of this study show that the performance of the porous plug type trapping device is influenced by external parameters, i.e., $\Delta T$ across the plug and the type of material that the plug is fabricated from. For plug type trapping devices, the two most important features are the maximum sustainable internal pressure or bubble point and the influence of controllable and uncontrollable parameters on the evaporation flux across the plug. Controllable parameters are the pore diameter and length, number of pores, the plug material, and back pressure conditions. The uncontrollable parameters are the nondimensional groupings that are dependent on the specific cryogen, e.g., liquid hydrogen.

The analysis showed that the evaporation flux is strongly dependent on the temperature differential across the pore and the pore surface temperature distribution. For Marangoni numbers representative of liquid hydrogen at the various superheated conditions, no significant effect on the surface temperature was observed. The evaporation flux increased primarily due to the reduction of $R s$. It was also observed that the increase in Marangoni number caused the dynamic pressure in the corner regions to decrease due to liquid being driven toward the center. This causes the dynamic pressure in the center to increase, thus increasing the curvature of the surface and decreasing the effective bubble point of the pore. As shown in figure 17, the increase in surface curvature is small, thus resulting in a small decrease in bubble point.

The effect of Biot number is most evident in the surface temperature distribution. As $B i$ increased, the surface temperature decreased, thus strongly influencing the evaporation across the pore. Although $B i$ caused a reduction in evaporation, it also reduced the strength of the surface thermal gradient, which reduced the thermocapillary convection and, subsequently, the dynamic pressure within the pore. This lessened the effect of dynamic pressure on the effective bubble point of the pore.

An important outcome of this study is the ability to numerically analyze the thermal distribution and flow field within the mircopores of the overall device. Previous empirical analysis on plug type trapping devices have measured the bubble point and evaporation rate for the specific plug tested on a macroscopic scale since no detailed analytical tool was available for performance predictions. As an example, assuming that a porous plug with a surface area of $4.9 \mathrm{~cm}^{2}$ and a porosity of 30 percent was installed into the wall of a liquid hydrogen dewar. Assuming a $\Delta T=1.0 \mathrm{~K}$ and accounting for 
thermocapillary convection, the total evaporation flow rate across the plug would be approximately $0.01665 \mathrm{gm} / \mathrm{s}$.

\section{Recommendations}

1. Porous Plug Applications. Based on the results discussed, the design of the porous plug should be driven by the application. If maximum evaporation flux across the plug is desired, the use of a highly conductive material for the plug material is recommended. This reduces the side boundary $B i$, thus transporting the maximum heat from the liquid to the free surface where evaporation is free to occur. On the other hand, if the plug is to be used as a phase separator for liquid acquisition, the use of a material that has a low thermal conductivity is recommended. This would serve to decrease the free surface thermal gradient, thus decreasing the thermocapillary convection and dynamic pressure and minimizing the decrease in bubble point.

The use of uniform cylindrical pores for all applications is desirable due to the simple, predictable, and repeatable geometry. This enables detailed microscopic analysis and allows the results to apply to similar plugs operated under identical conditions.

2. Future Research. The analysis performed in this study was limited in several ways. The following enhancements would add fidelity to the analysis.

(a) The validation of these results through experimentation. Simple test cases can be designed which could serve to confirm the results presented in this study. Macroscopic tests can be used to verify the evaporation across the plug. With the number of pores known, the evaporation across each pore can be determined. To evaluate the flow field distribution, a single, two-dimensional pore can be used with either liquid hydrogen or water with the relevant parameters adjusted by using chemical additives. The use of a microscope can be helpful in the flow field visualization. This will also serve to assess the accuracy of this model.

(b) The expansion of the analysis to three dimensions. This would more accurately model the actual physical geometry of the pore.

(c) A closer and more extensive examination of the effect of pressure differential across the pore on the resulting temperature and flow fields.

(d) Allowing the interface to translate within the pore depending on the applied pressure differential and the energy balance across the pore. This would serve to address previous studies which suggest that the interface will retreat into the pore with increasing $\Delta T$ and evaporation, i.e., pore dry out. This would also require that the transient solution be solved.

\section{E. Summary}

In this section, the results for the case of pure evaporation, combined evaporation/thermocapillary flow, and combined flow with a sidewall temperature gradient are discussed. The results seem to indicate that the material chosen for the fabrication of the plug should be application dependent. It was shown that a material with a high thermal conductivity, i.e., low $B i$, serves to increase the free surface temperature, thus increasing the evaporation. Therefore, a plug fabricated out of a this material would be best used for an application where heat rejection or vapor generation is required. On the other 
hand, if phase separation or liquid acquisition is required, a material with a high $B i$ should be used. This would serve to decrease the effect of the liquid dynamic pressure on the plug's bubble point.

In addition, several recommendations for the continuation of this study in terms of further analysis and experimentation are presented. 


\section{APPENDIX A}

\section{FREE SURFACE SOLUTION METHOD}

\section{A. Introduction}

In order to calculate the free surface position the nonlinear differential equation shown in section IV, equation (46), with the associated boundary conditions is solved using Newton's method for nonlinear systems. The methodology as it is applied to this problem is described in this section.

\section{B. Newton's Method for Nonlinear Systems}

5:

In order to calculate the free surface position, the coordinate system defined previously in figure

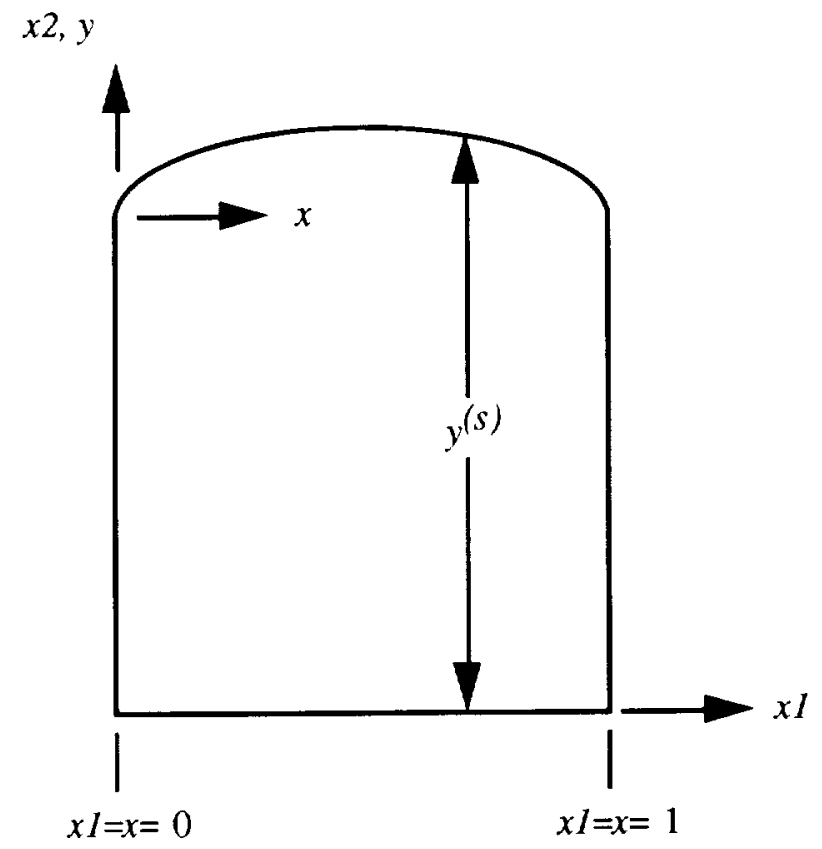

Figure A.1. Free surface coordinate system.

where $x=x 1, y=y^{(s)}-x 2$, and $y^{(s)}$ is the surface coordinate referenced from the bottom of the cavity.

Recalling the relations which defines the surface shape, namely, equation (46) and associated boundary conditions:

$$
\begin{gathered}
y_{, x x}-\left(\frac{B o y^{(s)}+\Pi}{1-D}\right)\left[1+\left(y_{, x}\right)^{2}\right]^{3 / 2}=0, \\
(y)_{x=0,1}=0, \\
\left(y_{, x}\right)_{x=1 / 2}=0,
\end{gathered}
$$


and expressing equation (A.1) in finite difference form:

$$
\frac{y_{i+1}-2 y_{i}+y_{i-1}}{h^{2}}-\left(\frac{B o y_{i}+\Pi}{1-D}\right)\left[1+\left(\frac{y_{i+1}-y_{i-1}}{2 h}\right)^{2}\right]^{3 / 2}=0 \text {, }
$$

Newton's method can be applied.

The approach that is used to solve for the surface is to use Newton's method for nonlinear systems to converge on a solution for the independent variable $y_{i}$. Newton's method employs the following iteration algorithm:

$$
y_{i}^{(k)}=y_{i}^{(k-1)}-\left(J_{i j}^{(k-1)}\right)^{-1} F_{j}^{(k-1)}
$$

where $J_{i j}$ is defined as the jacobian of $F_{i}$, and is given by:

$$
J_{i j}=\frac{\partial F_{i}}{\partial y_{j}}=\left[\begin{array}{ll}
\frac{\partial F_{1}}{\partial y_{1}} & \frac{\partial F_{1}}{\partial y_{2}} \\
\frac{\partial F_{2}}{\partial y_{1}} & \frac{\partial F_{2}}{\partial y_{2}}
\end{array}\right],
$$

and $F_{i}$ is defined as the root form of equation (A.4), namely:

$$
F_{i}=y_{i+1}-2 y_{i}+y_{i-1}-h^{2}\left(\frac{B o y_{i}+\Pi}{1-D}\right)\left[1+\left(\frac{y_{i+1}-y_{i-1}}{2 h}\right)^{2}\right]^{3 / 2} \text {, }
$$

where $h$ is the separation distance between nodes, $k$ is the iteration number and $i, j$ are tensor index values. Expressing equation (A.7) in the general form:

$$
F_{i}=y_{i+1}-2 y_{i}+y_{i-1}-h^{2} f\left(x_{i}, y_{i}, y_{i, i}\left(y_{i+1}, y_{i-1}\right)\right)
$$

the Jacobian can be found. obtained:

Equation (A.8) is differentiated with respect to $y_{i}$ and the following relation for the Jacobian is

$$
J_{i j}=\frac{\partial F_{i}}{\partial y_{j}}=\frac{\partial y_{i+1}}{\partial y_{j}}-2 \frac{\partial y_{i}}{\partial y_{j}}+\frac{\partial y_{i-1}}{\partial y_{j}}-h^{2} \frac{\partial f}{\partial y_{j}}
$$

Letting $\partial y_{i} / \partial y_{j}=\delta_{i, j}$, equation (A.9) can be written as, 


$$
J_{i j}=\delta_{i+1, j}-2 \delta_{i, j}+\delta_{i-1, j}-h^{2} \frac{\partial f}{\partial y_{j}}
$$

where $\delta_{i, j}$ is the Kronecker delta and is defined as having a valve of 1 when $i=j$ and 0 when $i \neq j$.

In order to determine $\partial f / \partial y_{j}, f$ is expressed as:

$$
f\left(x_{i}, y_{i}, y_{i}^{\prime}\left(y_{i+1}, y_{i-1}\right)\right)=f\left(x_{i}, h\left(y_{i}\right), g\left(y_{i}\right)\right),
$$

where,

$$
h\left(y_{i}\right)=y_{i},
$$

and

$$
g\left(y_{i}\right)=\frac{y_{i+1}-y_{i-1}}{2 h} .
$$

Using equations (A.11), (A.12), and (A.13), $\partial f / \partial y_{j}$ can be expressed as:

$$
\begin{gathered}
\frac{\partial f}{\partial y_{j}}=\frac{\partial f}{\partial h} \frac{\partial h}{\partial y_{j}}+\frac{\partial f}{\partial g} \frac{\partial g}{\partial y_{j}}, \\
=\frac{\partial f}{\partial h} \frac{\partial y_{i}}{\partial y_{j}}+\frac{\partial f}{\partial g} \frac{\partial}{\partial y_{j}}\left(\frac{y_{i+1}-y_{i-1}}{2 h}\right), \\
=\frac{\partial f}{\partial h} \delta_{i, j}+\frac{1}{2 h} \frac{\partial f}{\partial g}\left(\delta_{i+1, j}-\delta_{i-1, j}\right),
\end{gathered}
$$

and the expression for $J_{i j}$ can be written as:

$$
J_{i j}=\delta_{i+1, j}-2 \delta_{i, j}+\delta_{i-1, j}-h^{2}\left[\frac{\partial f}{\partial y_{i}} \delta_{i, j}+\frac{1}{2 h} \frac{\partial f}{\partial y_{i}^{\prime}}\left(\delta_{i+1, j}-\delta_{i-1, j}\right)\right] .
$$

By inspection of equation (A.17), $J_{i j}$ will be nonzero only in the range of $i-1 \leq j \leq i+1$. The nonzero values of $J_{i j}$ are given below:

$$
\begin{array}{ll}
j=i-1: & J_{i j}=1+\frac{h}{2} \frac{\partial f}{\partial y_{i}^{\prime}}, \\
j=i: & J_{i j}=-2-\frac{h}{2} \frac{\partial f}{\partial y_{i}}, \\
j=i+1: & J_{i j}=1-\frac{h}{2} \frac{\partial f}{\partial y_{i}^{\prime}},
\end{array}
$$

where $f$ is given by: 


$$
f=\left(\frac{B o y_{i}+\Pi}{1-D}\right)\left[1+\left(y_{i}^{\prime}\right)^{2}\right]^{3 / 2}
$$

Differentiating $f$ with respect to $y_{i}$ and $y_{i}^{\prime}$, results in the following:

$$
\frac{\partial f}{\partial y_{i}}=\left(\frac{B o}{1-D}\right)\left[1+\left(y_{i}^{\prime}\right)^{2}\right]^{3 / 2}
$$

and

$$
\frac{\partial f}{\partial y_{i}^{\prime}}=\left(\frac{B o+\Pi}{1-D}\right)\left[1+\left(y_{i}^{\prime}\right)^{2}\right]^{1 / 2} y_{i}^{\prime}
$$

Now solving for the root equation over the entire domain, it is observed that at $i=1$ and $i=N+1$, there are no values for $j=i-1$ and $j=i+1$, respectively, where $N=$ the number of intervals in the domain.

Considering the boundary conditions given in equations (A.2) and (A.3) for $i=1$ and $i=N+1$, respectively. Writing equation (A.7) at $i=2$, and applying the boundary condition given in equation (A.2), the following is obtained:

for $i=2$ :

$$
F_{2}=y_{3}-2 y_{2}+y_{1}-h^{2}\left(\frac{B o y_{2}+\Pi}{1-D}\right)\left[1+\left(\frac{y_{3}-y_{1}}{2 h}\right)^{2}\right]^{3 / 2} \text {, }
$$

and at $i=1$ :

$$
y_{1}=0
$$

therefore, equation (A.24) becomes:

$$
F_{1}=y_{2}-2 y_{1}-h^{2}\left(\frac{B o y_{1}+\Pi}{1-D}\right)\left[1+\left(\frac{y_{2}}{2 h}\right)^{2}\right]^{3 / 2}
$$

Now, considering the boundary condition at $i=N+1$ :

$$
F_{N+1}=y_{N+2}-2 y_{N+1}+y_{N}-h^{2}\left(\frac{B o y_{N+1}+\Pi}{1-D}\right)\left[1+\left(\frac{y_{N+2}-y_{N}}{2 h}\right)^{2}\right]^{3 / 2},
$$

and imposing the boundary condition at $i=N+1$ :

$$
y_{, x}=\frac{y_{N+2}-y_{N}}{2 h}=0
$$

or $y_{N+2}=y_{N}$, equation (A.27) becomes: 


$$
F_{N+1}=2 y_{N}-2 y_{N+1}-h^{2}\left(\frac{B o y_{N+1}+\Pi}{1-D}\right)
$$

Therefore, $F_{i}$ is defined over the interval of $i=2$ to $i=N+1$. If one now shifts the indices over the interval of $i=1$ and $i=N$, the following is obtained:

at $i=1$ :

$$
F_{1}=y_{2}-2 y_{1}-h^{2}\left(\frac{B o y_{1}+\Pi}{1-D}\right)\left[1+\left(\frac{y_{2}}{2 h}\right)^{2}\right]^{3 / 2}
$$

at $i=2 \rightarrow N-1$ :

$$
F_{i}=y_{i+1}-2 y_{i}+y_{i-1}-h^{2}\left(\frac{B o y_{i}+\Pi}{1-D}\right)\left[1+\left(\frac{y_{i+1}-y_{i-1}}{2 h}\right)^{2}\right]^{3 / 2},
$$

at $i=N$ :

$$
F_{N}=2 y_{N-1}-2 y_{N}-h^{2}\left(\frac{B o y_{N}+\Pi}{1-D}\right)
$$

Using the above definitions for the coefficients of the Jacobian, equations (A.18), (A.19), and (A.20), and the root equation given in equations (A.30), (A.31), and (A.32), the independent variable $y$ is solved for by iteration, using the algorithm given in equation (A.5).

\section{Summary}

In this appendix, Newton's method for nonlinear equations that is used to solve for the free surface of the porous plug is discussed. Appendix B lists the numerical representation of this method. 


\section{APPENDIX B}

\section{MODIFIED COMPUTER CODE}

\section{A. Introduction}

In this section the modifications that were made to the reference model numerical method are shown. This section and reference 8 taken together represents the complete numerical model used in this study.

\section{B. Meniscus Subroutine}

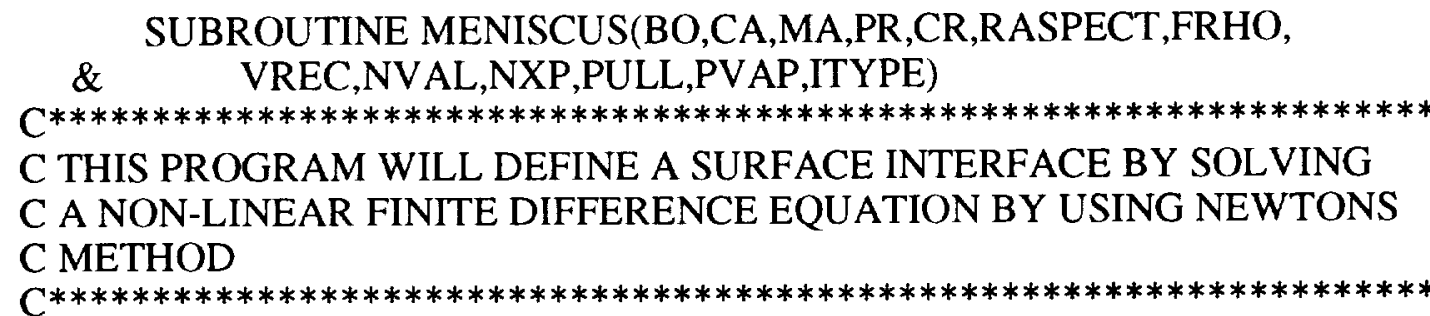

IMPLICIT DOUBLE PRECISION (A-H)

IMPLICIT DOUBLE PRECISION (O-Z)

$\mathrm{C}$

C CALCULATE ARRAY DIMENSIONS

$\mathrm{C}$

PARAMETER $(M X E=30, \quad M Y E=35)$

PARAMETER $(\mathrm{MXN}=1+2 * \mathrm{MXE})$

PARAMETER $(\mathrm{MYN}=1+2 * \mathrm{MYE})$

COMMON/SURFACE/

\& XP(MXN), YP(MXN), YSURFO(MXN),PD(MXN),PB(MXN),TEMP(MXN),

\& SURFNN(MXN,2),SURFNT(MXN,2)

$\mathrm{C}$

C DIMENSION ARRAYS AND DECLARE VARIABLES

$\mathrm{C}$

DIMENSION Y(MXN),TERM1(MXN),F(MXN),DEL(MXN),DY(MXN),R(MXN),

\& AJAC(MXN,MYN),TBAR(MXN),TERM2(MXN)

REAL MAIN

$\mathrm{C}$

C DEFINE FUNCTIONS

$\mathrm{C}$

FUNC1 $(\mathrm{X} 2, \mathrm{DYDX}, \mathrm{BOND}, \mathrm{CON} 1, \mathrm{CON} 2)=((\mathrm{BOND} * \mathrm{X} 2+\mathrm{CON} 1) /(1 .-\mathrm{CON} 2))^{*}$ $\&(1 .+\mathrm{DYDX} * * 2) * *$.

FUNC2(DYDX,BOND,CON2) $=(\mathrm{BOND} /(1 .-\mathrm{CON} 2)) *\left(1 .+\mathrm{DYDX}^{* *} 2.\right) * * 1.5$

FUNC3 $(\mathrm{X} 2, \mathrm{DYDX}, \mathrm{BOND}, \mathrm{CON} 1, \mathrm{CON} 2)=3 *((\mathrm{BOND} * \mathrm{X} 2+\mathrm{CON} 1) /(1 .-\mathrm{CON} 2))^{*}$

$\mathrm{C}$

$\& \operatorname{SQRT}(1 .+\mathrm{DYDX} * * 2). * \mathrm{DYDX}$

PHECEOHIO PAGE BLANK NOT FILMEO Pike Z 20 WTH 


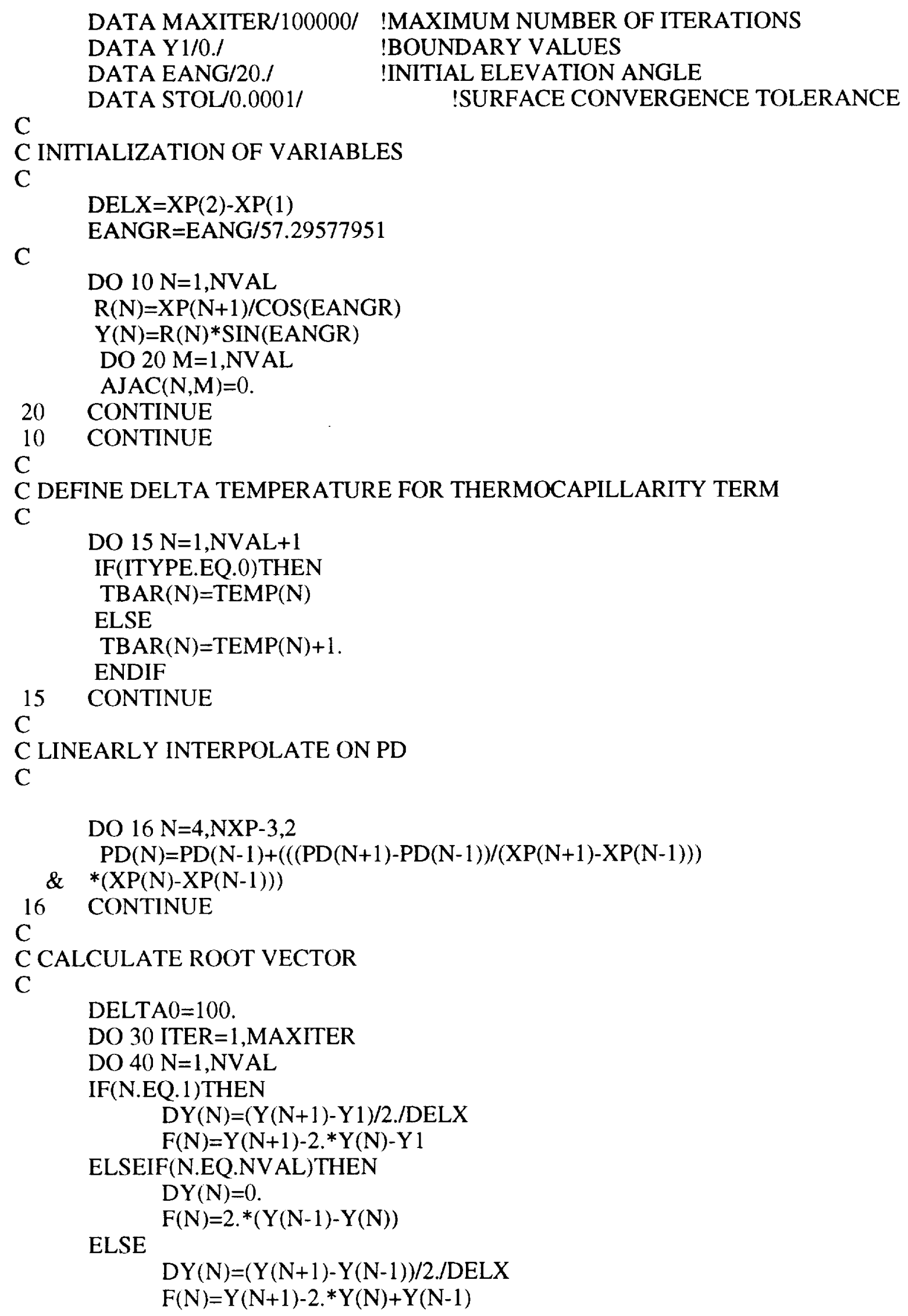




\section{ENDIF}

C

C CALCULATE SURFACE INFLUENCE DUE TO CA,PD,PULL,PVAP,VR,T,CR,TBAR

$\mathrm{C}$

$\operatorname{TERM} 1(\mathrm{~N})=-\mathrm{CA} *(\mathrm{PULL}+\mathrm{PD}(\mathrm{N}+1)-\mathrm{PVAP})+\mathrm{VREC} * \mathrm{TEMP}(\mathrm{N}+1) * * 2$.

$\operatorname{TERM} 2(\mathrm{~N})=\mathrm{CR} * \operatorname{TBAR}(\mathrm{N}+1)$

C

$\mathrm{F}(\mathrm{N})=\mathrm{F}(\mathrm{N})-\mathrm{FUNC} 1(\mathrm{Y}(\mathrm{N}), \mathrm{DY}(\mathrm{N}), \mathrm{BO}, \mathrm{TERM} 1(\mathrm{~N}), \mathrm{TERM} 2(\mathrm{~N})) * \operatorname{DELX} * * 2$.

40 CONTINUE

C

C CALCULATE THE JACOBIAN

$\mathrm{C}$

DO $50 \mathrm{~N}=1, \mathrm{NVAL}$

IF(N.NE.1) AJAC $(\mathrm{N}, \mathrm{N}-1)=1 .+\mathrm{DELX} *$

\& FUNC3(Y(N),DY(N),BO,TERM1(N),TERM2(N))/2.

AJAC $(N, N)=-2$.-FUNC2(DY $(N), B O, T E R M 2(N)) * D E L X * * 2$.

IF(N.NE.NVAL) AJAC $(\mathrm{N}, \mathrm{N}+1)=1$.-DELX*

\& FUNC3(Y(N),DY(N),BO,TERM1(N),TERM2(N))/2.

50 CONTINUE

C

C SOLVE FOR CHANGE IN Y-VALUE

C

C

CALL CROUT(AJAC,F,DEL,NVAL)

DELTA $=0$.

DO $60 \mathrm{~N}=1, \mathrm{NVAL}$

DELTA $=$ DELTA+DEL $(N)$

$\mathrm{Y}(\mathrm{N})=\mathrm{Y}(\mathrm{N})-\mathrm{DEL}(\mathrm{N})$

60 CONTINUE

C

IF(ABS(DELTA).LE.STOL)GOTO 500

DELTA $0=$ DELTA

C

30 CONTINUE

WRITE $(8,4000)$

4000 FORMAT(2X,'INTERFACE SOLUTION DID NOT CONVERGE',//)

$\begin{array}{ll}\text { STOP } \\ 500 & \text { CONTINUE }\end{array}$

C

C DETERMINE $X \&$ Y FOR RIGHT SIDE OF INTERFACE

C

$\mathrm{YP}(1)=\mathrm{Y} 1$

DO $65 \mathrm{~N}=2$, NVAL +1

$\mathrm{YP}(\mathrm{N})=\mathrm{Y}(\mathrm{N}-1)$

65 CONTINUE

$\mathrm{K}=0$

DO $70 \mathrm{~N}=\mathrm{NVAL}+2, \mathrm{NXP}$

$\mathrm{K}=\mathrm{K}+1$

$\mathrm{YP}(\mathrm{N})=\mathrm{YP}\left(\mathrm{N}-2^{*} \mathrm{~K}\right)$ 
CONTINUE

DO $75 \mathrm{~N}=1, \mathrm{NXP}$

$\mathrm{YP}(\mathrm{N})=\mathrm{YP}(\mathrm{N})+\mathrm{RASPECT}$

75 CONTINUE

DO $80 \mathrm{~N}=1, \mathrm{NVAL}$

DYDX $=(Y P(N+1)-Y P(N)) / D E L X$

$A L F A=D A T A N(D Y D X)$

SURFNN $(N, 1)=-1 .{ }^{*}$ DSIN(ALFA)

SURFNN(N, 2) $=$ DCOS (ALFA)

SURFNT $(\mathrm{N}, 1)=\mathrm{DCOS}(\mathrm{ALFA})$

80

SURFNT(N,2)=DSIN (ALFA)

SURFNN $($ NVAL $+1,1)=0$.

SURFNN(NVAL $+1,2)=1$.

SURFNT(NVAL $+1,1)=1$.

SURFNT(NVAL $+1,2)=0$.

$\mathrm{K}=0$

DO $85 \mathrm{~N}=\mathrm{NVAL}+2, \mathrm{NXP}$

$\mathrm{K}=\mathrm{K}+1$

SURFNN(N,1) $=-1 . *$ SURFNN $(\mathrm{N}-2 * \mathrm{~K}, 1)$

SURFNN(N,2) $=$ SURFNN $(\mathrm{N}-2 * \mathrm{~K}, 2)$

SURFNT $(\mathrm{N}, 1)=$ SURFNT $(\mathrm{N}-2 * \mathrm{~K}, 1)$

SURFNT(N,2)=-1. *SURFNT(N-2*K,2)

85 CONTINUE

RETURN

END

\section{Crout Subroutine}

SUBROUTINE CROUT(A,B,X,N)

$\mathrm{C} * * * * * * * * * * * * * * * * * * * * * * * * * * * * * * * * * * * * * * * * * * * * * * * * * * * * * * * * * * * * * * * * * * * *$

C SOLVES FOR THE X MATRIX USING A CROUT REDUCTION OF A NXN

C TRIDIAGONAL MATRIX, $\mathrm{AX}=\mathrm{B}$

$\mathrm{C} * * * * * * * * * * * * * * * * * * * * * * * * * * * * * * * * * * * * * * * * * * * * * * * * * * * * * * * * * * * * * * * * * * * *$

IMPLICIT DOUBLE PRECISION (A-H)

C

IMPLICIT DOUBLE PRECISION (O-Z)

PARAMETER (MXE $=30, \quad M Y E=35)$

PARAMETER (MXN $=1+2 * \mathrm{MXE})$

C

PARAMETER $(\mathrm{MYN}=1+2 * \mathrm{MYE})$

DIMENSION A(MXN,MYN),B(MXN),X(MXN),C(MXN,MYN),

C \& U(MXN,MYN),Z(MXN)

$\mathrm{NM} 1=\mathrm{N}-1$

C

C FIRST ROW 
C

$$
\mathrm{C}(1,1)=\mathrm{A}(1,1)
$$

$\mathrm{U}(1,2)=\mathrm{A}(1,2) / \mathrm{C}(1,1)$

C

C ROWS BETWEEN 1 AND N

C

$$
\begin{aligned}
& \text { DO } 10 \mathrm{I}=2, \mathrm{NM} 1 \\
& \mathrm{C}(\mathrm{I}, \mathrm{I}-1)=\mathrm{A}(\mathrm{I}, \mathrm{I}-1) \\
& \mathrm{C}(\mathrm{I}, \mathrm{I})=\mathrm{A}(\mathrm{I}, \mathrm{I})-\mathrm{C}(\mathrm{I}, \mathrm{I}-1) * \mathrm{U}(\mathrm{I}-1, \mathrm{I})
\end{aligned}
$$

10 CONTINUE

C

C LAST ROW

C

$$
\begin{aligned}
& \mathrm{C}(\mathrm{N}, \mathrm{N}-1)=\mathrm{A}(\mathrm{N}, \mathrm{N}-1) \\
& \mathrm{C}(\mathrm{N}, \mathrm{N})=\mathrm{A}(\mathrm{N}, \mathrm{N})-\mathrm{C}(\mathrm{N}, \mathrm{N}-1) * \mathrm{U}(\mathrm{N}-1, \mathrm{~N})
\end{aligned}
$$

C

C SOLVE FOR LZ=B

C

$$
\begin{aligned}
& \mathrm{Z}(1)=\mathrm{B}(1) / \mathrm{C}(1,1) \\
& \text { DO } 20 \mathrm{I}=2, \mathrm{~N}
\end{aligned}
$$$$
\mathrm{Z}(\mathrm{I})=(\mathrm{B}(\mathrm{I})-\mathrm{C}(\mathrm{I}, \mathrm{I}-1) * \mathrm{Z}(\mathrm{I}-1)) / \mathrm{C}(\mathrm{I}, \mathrm{I})
$$

20 CONTINUE

$\mathrm{C}$

C SOLVE FOR UX=Z

C

30 CONTINUE

$$
\begin{aligned}
& X(N)=Z(N) \\
& \text { DO } 30 I=1, N M 1 \\
& \mathrm{~K}=\mathrm{N}-\mathrm{I} \\
& \mathrm{X}(\mathrm{K})=\mathrm{Z}(\mathrm{K})-\mathrm{U}(\mathrm{K}, \mathrm{K}+1) * \mathrm{X}(\mathrm{K}+1)
\end{aligned}
$$

C

RETURN
END

\section{Sidewall Boundary Condition Modification}

C

C SET LEFT AND RIGHT BOUNDARY CONDITIONS

$\mathrm{C}$

IF(ITBC.EQ.0)THEN

$\mathrm{NL}=1$

$\mathrm{NR}=\mathrm{NXP}$

DO $157 \mathrm{~N}=1, \mathrm{NYP}$

$\operatorname{TEMP}(\mathrm{NL})=\mathrm{TSF}(1)$

$\operatorname{TEMP}(\mathrm{NR})=\mathrm{TSF}(1)$

$\mathrm{NL}=\mathrm{NL}+\mathrm{NXP}$

$N R=N R+N X P$

157 CONTINUE 


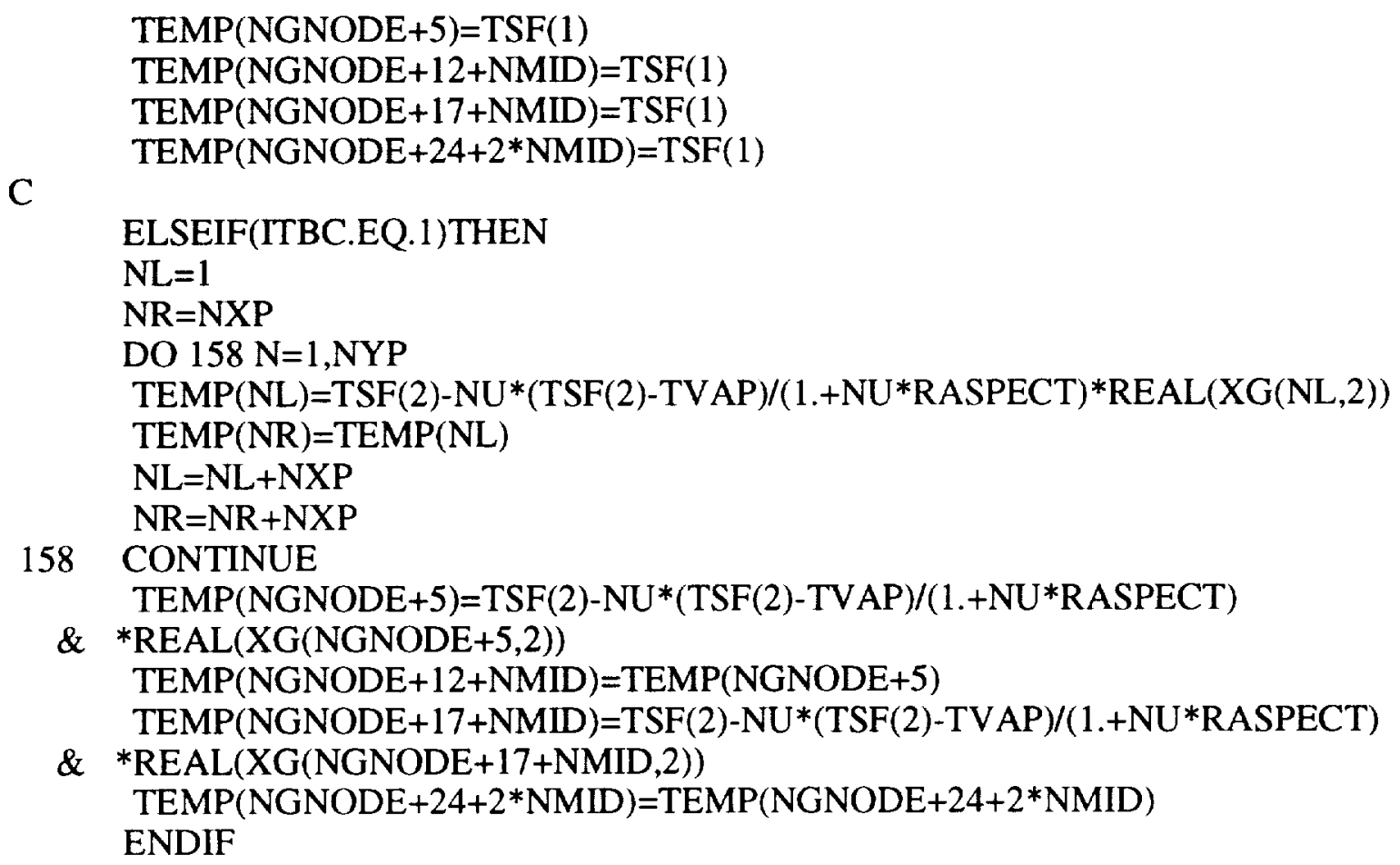

\section{E. Lower Boundary Condition Modification}

$\mathrm{UMAX}=(3 . / 2 .)^{*} \mathrm{QFLOW}$

$\mathrm{ACAV}=0.5$

DO 69 NBOT $=1$, NXP VELBOT $($ NBOT $)=U M A X *\left(1 .-\left((\operatorname{REAL}(\mathrm{XSURF}(\mathrm{NBOT}))-\mathrm{ACAV}) * * 2 . / \mathrm{ACAV}^{* *} 2.\right)\right)$ CONTINUE 


\section{REFERENCES}

1. Lin, C.S., et al.: "A Pressure Control Analysis of Cryogenic Storage Systems." AIAA Joint Propulsion Conference, AIAA Paper 91-2405, June 24-27, 1991.

2. Fazah, M.M., et al.: "Design and Integrated Operation of an Innovative Thermodynamic Vent System Concept." AIAA Joint Propulsion Conference, AIAA Paper 93-2427, June 28-30, 1993.

3. Sorensen, G.L., et al.: "A Zero-Gravity Thermodynamic Vent System for the Shuttle/Centaur Hydrogen Tank," Fourteen Intersociety Conference on Environmental Systems, SAE Paper 840974, July $16-19,1984$.

4. Karr, G.R., and Urban, E.W.: "Superfluid Plug as a Control Device for Helium Coolant." Cryogenics, vol. 20, 1980.

5. Frederking, T.H.K., et al.: "Sintered Plug Flow Modulation of Vapor-Liquid Phase Separator." Advances in Cryogenic Engineering, vol. 29, Plenum Press, New York, NY, 1984.

6. Hendricks, J.B., and Karr, G.R.: "Characterization of Superfluid Porous Plug Perfromance." Proc. Ninth Intl. Cryo. Engr. Conf., Butterworth, Guildford, UK, 1982.

7. Hendricks, J.B., and Karr, G.R.: "Superfluid Porous Plug Performance." Advances in Cryogenic Engineering, vol. 29, Plenum Press, New York, NY, 1984.

8. Schmidt, G.R.: "Thermocapillary Flow With Evaporation and Condensation and Its Effect on Liquid Retention in Low-G Fluid Acquisition Devices." A dissertation, The University of Alabama in Huntsville, Huntsville, AL, 1993.

9. Hendricks, J.B., and Karr, G.R.: "The Liquid-Vapor Transition in Porous Plug Operation." Advances in Cryogenic Engineering, vol. 31, Plenum Press, New York, NY, 1986.

10. Schotte, U.: "Mass Flow and Critical Velocity of Helium II in a Liquid Vapor Separating System." Z. Phys. B, 1982.

11. Hendricks, J.B. and Karr, G.R.: "Long Lifetime, Spaceborne, Closed Cycle Cryocooler." Alabama Cryogenic Engineering, Inc., Final Report, Contract NAS8-35254, April 1987.

12. Hendricks, J.B., and Dingus, M.L.: “A Phase Separator for Space Cryogen Systems.” Alabama Cryogenic Engineering, Inc., Final Report AFAL-TR-88-048, Contract F04611-87-C-0078, June 1988.

13. Sasmal, G.P., and Hochstein, J.I.: "Transient Analysis of Thermocapillary Convection in a Cavity." AIAA Aerospace Sciences Meeting and Exhibit, AIAA Paper 93-0917, January 11-14, 1993.

14. Kennard, E.: "Kinetic Theory of Gases." First Edition, McGraw-Hill, New York, NY, 1938.

15. Burden, R.L., and Faires, J.D.: "Numerical Analysis." Third edition, Prindle, Weber \& Schmidt, Boston, MA, 1985.

16. Fox R.W., and McDonald, A.T.: "Introduction to Fluid Mechanics." Wiley, New York, NY, 1985. 
17. Jaluria, Y., and Torrance, K.E.: “Computional Heat Transfer.” Hemisphere, Washington, DC, 1986.

18. Reynolds, W.C.: "Thermodynamic Properties in SI." Department of Mechanical Engineering, Stanford University, Stanford, CA. 


\title{
APPROVAL
}

\section{TRANSPORT PHENOMENA IN THE MICROPORES OF PLUG-TYPE PHASE SEPARATORS}

\author{
By M.M. Fazah
}

The information in this report has been reviewed for technical content. Review of any information concerning Department of Defense or nuclear energy activities or programs has been made by the MSFC Security Classification Officer. This report, in its entirety, has been determined to be unclassified.

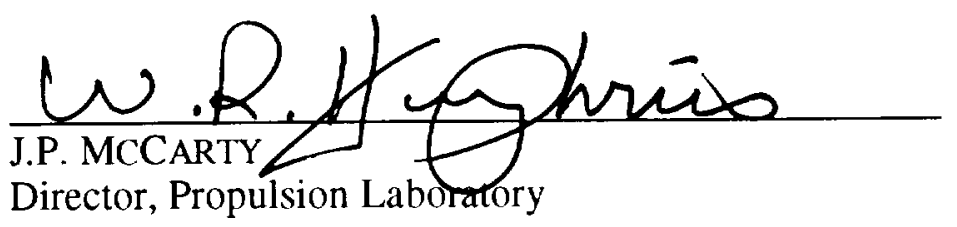


Puolic reporting burden for this colfection of information is estimated to average i hour per response. including the time for reviewing instructions. searching existing data sources. Jathering and maintaining the data needed, and completing and reviewing the collection of information. Send comments regarding this burden estimate or any other aspect of this

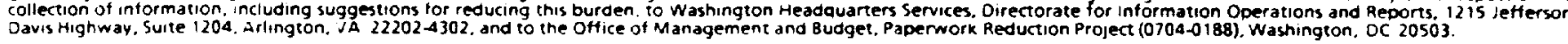
1. AGENCY USE ONLY (Leave blank)
2. REPORT OATE February 1995
3. REPORT TYPE AND DATES COVERED
Technical Memorandum

4. TITLE AND SUBTITLE

Transport Phenomena in the Micropores of

Plug-Type Phase Separators

\section{FUNDING NUMBERS}

6. AUTHOR(S)

M.M. Fazah

7. PERForming organization name(5) AND ADdRESS(ES)

George C. Marshall Space Flight Center

8. PERFORMING ORGANIZATION

Marshall Space Flight Center, Alabama 35812

National Aeronautics and Space Administration

Washington, DC 20546

10. SPONSORING / MONITORING AGENCY REPORT NUMBER

NASA TM-108479

11. SUPPLEMENTARY NOTES

Prepared by Propulsion Laboratory, Science and Engineering Directorate.

12a. DISTRIBUTION/AVAILABILITY STATEMENT

12b. DISTRIBUTION CODE

Unclassified-Unlimited

13. ABSTRACT (Maximum 200 words)

This study numerically investigates the transport phenomena within and across a porous-plug phase separator. The effect of temperature differential across a single pore and of the sidewall boundary conditions, i.e., isothermal or linear thermal gradient, are presented and discussed. The effects are quantified in terms of the evaporation mass flux across the boundary and the mean surface temperature. A two-dimensional finite element model is used to solve the continuity, momentum, and energy equations for the liquid.

Temperature differentials across the pore interface of $1.0,1.25$, and $1.5 \mathrm{~K}$ are examined and their effect on evaporation flux and mean surface temperature is shown. For isothermal side boundary conditions, the evaporation flux across the pore is directly proportional and linear with $\Delta T$. For the case of an imposed linear thermal gradient on the side boundaries, Biot numbers of $0.0,0.15$, and 0.5 are examined. The most significant effect of Biot number is to lower the overall surface temperature and evaporation flux.

14. SUBJECT TERMS

porous plug, phase separator, liquid acquisition, pressure control, surface tension

15. NUMBER OF PAGES 59

16. PRICE COOE NTIS

\begin{tabular}{|c|c|c|}
\hline $\begin{array}{l}\text { 17. SECURITY CLASSIFICATION } \\
\text { OF REPORT }\end{array}$ & $\begin{array}{l}\text { 18. SECURITY CLASSIFICATION } \\
\text { OF THIS PAGE }\end{array}$ & $\begin{array}{l}\text { 19. SECURITY CLASSIFICATION } \\
\text { OF ABSTRACT }\end{array}$ \\
\hline Unclassified & Unclassified & Unclassi \\
\hline
\end{tabular}

20. LIMITATION OF ABSTRACT

Unlimited

Standard Form 298 (Rev 2-89) 